\section{Index of abstract keyword}

11C-L-methionine PFU-1

3 Tesla PNE-20, PNE-24

$6 p$ deletion PMS-11

abdomen RS-5, PFN-17, EM-11

abnormality, congenital GU-9,

PFN-13, FN-R1

abnormality, rachitic PMS-53

abruption, placenta PFN-14

abscess, epidural PMI-23

abscess, musculoskeletal PMS-19

abscess, periappendiceal PIR-6

abscess, retropharyngeal PNE-7

accidental injury MS1-R1

acoustic radiation force impulse

EM-1, EM-3

acquired PGI-34

acquisition time NE1-R1

acute scrotum PGU-18, PGU-22

adaptive statistical iterative

reconstruction RS-4, PRS-9, RS-5,

PRS-17, PRS-22

adolescent PGU-2, PRS-3, PON-24,

PGI-56, GU-R2

adrenarche PGU-20

aganglionosis, total colonic PGI-12

agenesis, uterine PGU-39

agenesis, vaginal PGU-39

ALARA PGI-16, RS-14, MS2-10

algorithm PMI-24

alignment PMS-36

alkaline phosphatase

alveolus $\mathrm{CH}-1$

ambiguous genitalia PGU-24

american board of radiology, residency

training PED-1

amniotic band PFN-24

amoebic PNE-15

amputation PMS-58

anaesthesia PMI-2

analysis GI-14

anatomy of gastro oesophageal

junction PGI-32

aneurysm PNE-62 aneurysm, aorta PMI-32

angiitis PNE-68

angiography PCV-9, PGU-40

angiomatoid fibrous histiocytoma

PMS-56

animal model FU-5

anisotropy PNE-69

ankle MS2-R1

anomaly, branchial PNE-18

anomaly, facial, midline PNE-38

anomaly, lung, congenital $\mathrm{CH}-7$

anomaly, Müllerian PGU-38

anomaly, spine PNE-49

anomaly, vascular PNE-62, MS2-14

antenatal diagnosis PFN-9

Antley-Bixler syndrom PNE-10

aorta PCV-8

aorto-carina-compression syndrome

$\mathrm{CH}-15$

aplasia, lung PCH-22

apparent diffusion coefficient PNE-12

appendage, testicular PGU-29, GU-10

appendagitis PGI-52

appendectomy, delayed PIR-6

appendicitis PGI-72, PRS-15, PGI-43, PGI-48, PGI-61

appendicitis, complication PIR-6

appendicitis, perforated PIR-6

appendix EM-6

arthritis PMS-33, FU-5

arthritis, inflammatory MS1-4

arthritis, juvenile PMS-16

arthropathy PMS-5, FU-5

arthroscopy PMS-62

aspergillosis PMI-23

Aspergillus PCH-4

aspiration, fine-needle IR-5

asplenia syndrome $\mathrm{CV}-7$

atresia, biliary GI-9

atresia, bronchial $\mathrm{CH}-14$

atresia, duodenal PGI-23

atrophy, kidney PGU-27

atrophy, muscle PMS-30

attitude, physician's FN-R1

auto-inflammatory disorder PMI-21 automatic tube current

modulation RS-9

automotive safety PFN-14

autopsy PFN-9, PMI-26, EM-12

avascular necrosis MS1-5

balloon burst IR-3

balloon occlusion PCH-13

bariatric PGI-56

barium enema GI-16

Bartonella henselae PMI-25

battery, button PGI-68

best practice model PMI-12

bile duct, common PIR-3

biliary atresia PGI-20, PGI-24, PGI-44

biliary atresia, extrahepatic GI-10

biliary tract PGI-36

biomarker EM-10

birth MS1-1

birthmark PMI-5

bismuth shield RS-9

bladder extrophy, classic PGU-32

blood FU-5

bone NE2-R1, MS2-R2, PMS-47,

PFN-19

bone disease, metabolic PMS-49

bone scintigraphy EM-R3

bone, long NA-5

bone, lytic lesion PON-27

botulinum toxin PNE-40

bowel, see intestine

bowel wall thickness GI-3

brachial plexus MS1-1

brachial plexus birth injury PMS-12

brain NE2-R1, PNE-6, FN-R2, NE1-4,

FN-2, NE1-5, RS-9, PNE-21, PFN-6,

PNE-26, PFN-11, PFN-13, PNE-45,

PFN-17, PNE-66, FU-6

brain death PNE-52

brain lesion PFN-2

brain perfusion NE1-12

brainstem PNE-2

breast shield RS-10

broncho-pulmonary sequestration

PCH-18 
bronchography $\mathrm{PCH}-13$

bronchopulmonary dysplasia, see chronic lung disease of prematurity bronchopulmonary sequestration PCH-10

bronchoscopy $\mathrm{PCH}-12$

bronchus, tracheal CV-7

bufford PMS-62

Burkit lymphoma

calcifying epithelioma of Malherbe PMS-51

callosotomy NE1-8

calyectasis GU-4

cannulation, venous MI-1

capsule PGU-34

carcinoma, midline, NUT PON-35

cardiomyopathy $\mathrm{CV}-11$

cardiovascular EM-R1

cartilage PMS-37

cat scratch disease PMI-25

catheter, umbilical FN-10

central nervous system PNE-34

central review ON-4

cerebellum FN-11, PFN-25

cerebral palsy NE1-R2

cerebrospinal fluid flow EM-4

cerebrovascular reactivity NE1-14

cervical spine PMS-11

chest PCH-5, PON-13, CH-R2,

FN-6, CH-12

chest drain PIR-2

chest wall PON-12, PON-13

chickenpox PMI-9, PNE-2

child abuse NA-1, NA-3, NA-R2,

PNE-22, PMS-31, PMS-63, NA-5,

NA-4, NA-R1

cholestasis, neonatal GI-9

cholesteatoma PNE-18, PNE-23

choral hydrate PMI-19

chronic lung disease of prematurity

EM-14, CH-4, CH-5, CH-1

cine CV-4, EM-4, FN-4

classification PMS-20, MS2-4

cleft lip and palate FN-4

clinical trial MS2-R1

cloacal extrophy PFN-4

club foot

coalition MS2-12

cochlea PNE-14

coil EM-11

coil, customised NE1-2

collateral, portosystemic PGI-24,
PGI-26

collecting system GU-4

coloboma PNE-37

colour Doppler GI-6

colour fraction PMS-16

communication PMI-27, PMI-28,

PMI-27

compartment syndrome PMS-30

complication PMI-9, PMI-20, PNE-46

complication, surgical PNE-36

compressed sensing PMS-44

compressed-sensing CV-10

computed tomography PGU-1, PRS-3, EM-R2, PGI-70, PNE-9, PGI-7,

PRS-6, PCH-5, RS-4, MI-1, PRS-9, PRS-10, PON-15, PFN-5, RS-6,

ON-2, PNE-16, RS-9, PMI-12,

PGU-14, PCH-7, RS-3, RS-R1,

PCH-13, CH-10, PCH-16, PCH-17,

PCH-20, PCH-21, PGI-35, PCV-7,

PNE-37, PRS-15, PMI-25, PNE-39,

PMI-26, PNE-45, PON-31, PGI-47,

PNE-59, PON-36, PON-38, PNE-64,

PRS-17, NE1-11, CH-R2, PRS-19,

CV-9, RS-R2, RS-R3, RS-8, RS-12,

EM-12, CH-12, PGI-61, RS-1, ON-8,

PMI-36, CH-14

computed tomography angiography

PCV-1, PIR-7, RS-10, CH-14

computed tomography angiography, cardiac $\mathrm{CH}-13$

computed tomography angiography, chest $\mathrm{CH}-13$

computed tomography, abdomen PRS-2 computed tomography, chest PRS-1,

PCH-12

computed tomography, dose reduction

RS-7

computed tomography, head PNE-8,

PNE-72

computed tomography, high-resolution

CH-R3, PCH-6, CH-5

computed tomography, micro- MS1-4

computed tomography, multi-detector

row CH-8, PCV-3, PCV-4, RS-5,

PGI-26, PON-26, PMI-32, CH-7

computed tomography, peripheral

MS1-4

computed tomography, radiation dose

PRS-8, RS-7

computed tomography, ultrafast $\mathrm{CH}-13$

computed tomography, volumetric, low-dose PNE-33 congenital PCH-5, PGU-16, PNE-26, PGI-34, PMS-55

connectivity, functional NE1-7

constrictive bronchiolotis $\mathrm{CH}-10$

contrast agent, ultrasound, second

generation GU-7

contrast medium GU-8, PMI-22

contrast medium, iodinated PRS-6

contrast medium, magnetic resonance imaging CV-8, PON-28, PNE-56,

PCV-9

contrast medium, oral PGI-63

contrast-enhanced ultrasound PGI-5

contrast-enhanced, late $\mathrm{CV}-8$

contrast-enhancement, magnetic

resonance imaging, late PCV-2

conus medullaris PNE-3

conversion coefficients RS-3

coronary artery, aberrant CV-R1

coronary artery, anomalous aortic

origin of CV-3

coronary vessels, anomaly PCV-3

corpus callosum PFN-6

cost-effectiveness PFN-21

cost-saving PMI-18

counselling, prenatal FN-R1

cranial RS-R3

craniocervical stenosis PNE-62

craniofacial PNE-59

craniosynostosis NE1-12

Crohn disease EM-9

Crohn diseases PGI-3, PGI-4, EM-8,

GI-17

cross-sectional imaging CV-3

curriculum, paediatric radiology PED-1

cyst PCH-2, PNE-35

cyst, bone IR-4

cyst, central nervous system PFN-13

cyst, ganglion PMS-3

cyst, mesenteric PGI-52

cyst, ovary PFN-20

cyst, urachus PGI-52

cystic fibrosis EM-1, PGU-11, EM-13

cystography, positional installation of

contrast PGU-8

cystoscopy PGU-19

cytomegalovirus infection, congenital PFN-2

deceased PMS-8

defect, heart CV-2

dehydration PGU-11

dermal sinus tracts, spinal PNE-13 
dermoid, nasal PNE-38

development, neuro- PFN-23

developmental MS2-3

developmental dysplasia of the hip

PMS-2, MS2-2, MS2-5, PMS-20,

MS2-4, MS2-1, MS2-6

diagnosis GI-6, PGI-6, PGI-7, CH-3, PGI-21

diagnosis, missed PMS-30

diagnosis, prenatal PFN-8

dialysis, peritoneal PGU-34

difficult news PMI-28

diffusion tensor imaging NE1-3,

PNE-11, NE1-9, PNE-65, PNE-67,

NE1-R1, NE1-8, NE1-13, PFN-25

digital infrared thermal imaging MS1-6

disease, systemic PNE-57

dislocation, hip PMS-2

disorders of sexual development

PGU-24

dissection PNE-62

diuretıc PGU-4

dog-attack PCH-20

Doppler PGI-13, PMS-16, PGI-20,

GI-7, PGU-40

Doppler ultrasound PMI-32

Doppler, colour PGI-40

Doppler, pulsed

Doppler, transcranial PNE-42, PNE-52

dose reduction PRS-2, PRS-9,

PRS-11, RS-5, FU-3

dosimetry PRS-6

double bubble-sign PGI-23

doxycycline PIR-8

drainage, percutaneous PIR-3

drug-safety PMI-22

duodenal compression GI-15

duodenum PGI-21

duplication cyst PGI-21

dysostosis PFN-9

dysplasia MS2-3

dysplasia, focal cortical PNE-54,

PNE-55

education PED-3

effectiveness PMI-15

efficacy, diagnostic MS1-6

effusion PIR-2

effusion, loculated $\mathrm{PCH}-8$

effusion, pleural $\mathrm{PCH}-14$

elastography EM-3, EM-2

elbow PMS-39, PMS-40, PMS-43,

MS2-R1 embolisation PIR-7

embolisation, Onyx IR-1

embolism, pulmonary $\mathrm{PCH}-21$

embryogenesis PNE-45

emergency PGI-73, PGI-45

emphysema, congenital lobar PCH-22

empyema PIR-2

encephalitis PNE-1, PNE-28

encephalocele PNE-38

encephalopathy PNE-51

endocrinology PGU-24

endometriosis PGU-39

endoscopy, neuro- PNE-46

enterocolitis, necrotising GI-6

enterography PGI-4

enterolith PFN-16

enterovirus PNE-1

epidermoid PNE-38

epilepsy PNE-11, NE1-9, PNE-44,

PNE-54, PNE-55, PNE-63, NE1-7

epilepsy, refractory PNE-24

epiphysis PMS-30

epiphysis, upper femoral,

slipped PMS-28

epithelioid haemangioendothelioma

PON-4

equipment PMI-8

error PED-2

examination, physical

exposure control, automated RS-6

extra-corporeal membrane

oxygenation PCV-1

extremity PON-25, MS2-11

extremity lesion PMS-32

eye PNE-37

eyelid PON-23

family-centred care PMI-27, PMI-27

fat GI-15, PGI-2

fat fraction GI-12

fat, brown FU-1

fatal PMI-21

feasibility PMI-31

feed and wrap PMI-14

femur PON-4

fever PMI-21, PFU-2

fibromatosis PON-21, PNE-39

fibrosis EM-2

filtered back projection PMI-36

findings, radiography $\mathrm{PCH}-11$

finite element model PFN-14

fistula PGI-68

fistula, perianal GI-17 fistula, tracheo-oesophageal PGI-10

flouroscopy PMI-30

flow CV-10

fluoro-deoxy-glucose FU-3, FU-1

fluoroscopy PRS-13, PED-4

fluoroscopy, digital pulse

fluoroscopy, upper gastrointestinal

tract PGI-42, GI-19

focal nodular hyperplasia ON-10

foetus PMI-1, PNE-3, PNE-4, CV-2,

FN-R2, FN-2, PFN-4, FN-7, PCH-13,

PFN-6, PFN-8, PFN-11, PFN-16,

PFN-19, PON-34, CV-1, FN-1, FN-6,

FN-5

foetus, demise PFN-14

foetus, heart PFN-7

foetus, karyotype FN-14

foetus, marker FN-14

follow-up CH-3, PGI-20

foot PMS-36

foot alignment PMS-29

foreign body PGI-68

foreign body, oesophagus

four-dimensional EM-R1

fracture PMS-13, NA-3, MS1-R1,

PMS-43, PMS-63, NA-5, MS1-6

fracture dating NA-1, NA-R1

fracture healing NA-1

functional imaging, lung EM-14

fungus PGU-13

gadofosveset trisodium PNE-56

gadoxetic acid PON-28

gallbladder PGI-36

gastrointestinal PGI-11, PFN-16

gastrointestinal congenital

abnormalities PGI-71

gastroschisis PFN-4

gate-keeping PMI-12

general anaesthesia PMI-14

genetic PMI-5

genital anomalies, female GU-1

genitography PGU-24

glioma, nasal PNE-38

Gorlin PFN-10

gradient-echo PNE-66

granuloma, eosinophilic PON-27

grey matter NE1-6

groin PMI-7

guidelines PMI-13, PMS-31

guidelines, National Institute of

Clinical Excellence PNE-8

gynaecology GU-R2 


\section{H1N1 PCH-11}

haemangioma PGI-14, MS2-14

haemangioma, infantile ON-9

haemophilia MS2-R1, FU-5

haemorrhage PNE-2, FN-11, FN-12,

PIR-7

haemorrhage, adrenal ON-R1

haemorrhage, brain PFN-13

haemorrhage, intracranial PNE-46

haemorrhage, intraventricular PFN-22

haemorrhage, spinal PNE-22

Haller index CH-11

hand MS2-9, PMS-55

handlebar hernia PGI-47

harness MS2-3

head RS-R3

head and neck RS-8

headache PNE-72

health care costs PGI-67

hearing loss PNE-14

heart CV-1, CV-9, CV-10

heart disease, congenital CV-4, CV-8,

PFN-7, PCV-8, CV-6, CV-R1

heat stroke PNE-17

hemihypertrophy ON-3

hemihypertrophy, isolated ON-3

hemiplegia PNE-43

hemispherotomy NE1-9

hepatic fibrosis EM-3

hepatobiliary PGI-9

hepatoblastoma ON-R2, PON-38

hepatocellular carcinoma PON-38

hernia PMS-67

hernia, diaphragma, congenital

PCH-22, FN-5

hernia, inguinal PGU-9, PGI-29

hernia, internal PGI-52

high-resolution $\mathrm{PCH}-15$

highly active antiretroviral therapy

CH-9

hindfoot PMS-41, MS2-12

hip MS2-2, MS2-3

hip, development MS2-7

hippocampus, hyperintensity

PNE-17

Hirschsprung allied disease GI-16

Hirschsprung disease GI-16

histiocytosis PNE-64

HIV PCH-4, PCH-7

holoprosencephaly PFN-6

hyaline membrane disease $\mathrm{CH}-3$

hybrid imaging EM-R3

hydrocephalus PNE-46 hydronephrosis GU-2, GU-4

hydronephrosis, antenatal PFN-21

hydroureter GU-4

hyoscine butylbromide PGI-63

hyperplasia, focal nodular ON-10

hypertension PGU-40

hypertension, portal GI-8

hypertension, renovascular PGU-40

hypophosphatasia

hypoplasia, pulmonary $\mathrm{PCH}-22$

hypospadias PGU-6

hypothyroidism, congenital PMI-33

hypoxia-ischaemia PFN-17, FN-9

hypoxia, perinatal NE1-4

iDose PMI-36

image interpretation, chest

radiography $\mathrm{PCH}-1, \mathrm{PGU}-32$

image noise PRS-22

image processing $\mathrm{PCH}-17$, GU-5

image quality PRS-6, PRS-9, PRS-11,

RS-R1

immobilisation, see restraint

immunocompromise PCH-4

impairment, neurological PGI-67

impingement, femoroacetabular MS2-7

in-patient PMI-31

indication RS-R3

infant FN-11, PON-9, PMS-11,

CH-R3, GU-7, PGU-9, PNE-12,

PGU-11, PCH-16, PON-22, CH-R2,

$\mathrm{CH}-5$

infarct FN-12

infarct, spleen PGI-25

infection MI-2, PMS-19, PMI-17,

PMS-38, PGI-39

infection, chest PCH-4

infection, lower respiratory tract $\mathrm{PCH}-1$

infection, urinary tract GU-6

infection, varicella-zoster PNE-28

inflammation PMS-38, PMS-47, FU-5

inflammatory bowel disease EM-7,

PGI-51, PGI-58, GI-5, GI-4

infratemporal PON-23

injection, shoulder MS2-10

injury PGI-5, MS2-9, PMS-39,

PFN-11, PGU-28, PMS-43, PFN-14,

PMI-35, RS-R3, PMS-66

injury, acute PMS-39

injury, blunt PIR-3

injury, chronic PMS-39

injury, duodenum NA-R2

injury, head, blunt NE1-11 injury, mechanism of NA-3, MS1-R1

injury, visceral NA-R2, PGI-47

instability, atlantoaxial PNE-62

instrumentation PMS-65

intensive care NE1-2

intersex PGU-24

intestine EM-5, EM-7, PGI-19, FN-9, GI-5

intracranial lesion PNE-30

intraoperative NE1-1

intravenous contrast

intussusception PGI-1, PGI-16, PGI-18, GI-18, PGI-27, PGI-28, PGI-40

iron PNE-6, GI-13, GI-14

ischaemia, mesenteric PGI-52

isotropic EM-11

joint PMS-33, MS2-R1

justification PRS-7

Kawasaki disease PNE-7

ketamine MI-1

kidney PGU-4, FN-7, PGU-11, GU-4

kidney disease, chronic PGU-37

kidney disease, polycystic PGU-37

kidney, multicystic dysplastic GU-1, PGU-27

knee MS1-3, PMS-44, MS2-R1

knee joint FU-5

Langerhans cell histiocytosis CH-R3, PNE-21, PON-27

lap-band PGI-56

laser ablation, endovenous IR-2

lateral views NA-5

lawn mower PMS-58

leukoencephalopathy, cerebellar

PNE-64

line, indwelling PMI-20

liver EM-2, PON-22, PGI-35, PGI-39,

PGI-50, PON-38, GI-7, GI-13, GI-14

liver disease, diffuse PGI-13

liver disease, fatty, non-alcoholic

GI-12

liver mass $\mathrm{ON}-10$

liver transplant PGI-24, PGI-26

liver tumour PON-10

Loeys-Dietz PNE-62

long-term follow-up MS2-6

low-dose PNE-9

lumbar MS1-7

lumbar puncture PNE-4

lung PCH-2, PCH-12, PCH-24, EM-13 
lung clearance index $\mathrm{CH}-10$

lung disease, diffuse interstitial

PCH-6, PCH-24

lymph node GI-3

lymphadenopathy, neck PMI-16

lymphangioma, cystic PGU-22

lymphoblastic

lymphoma FU-4, ON-5, FU-3,

FU-1, FU-2

lymphoma, Hodgkin MS1-5,

ON-6, ON-4

lymphoma, non-Hodgkin PON-7

macrophage activation syndrome

PMI-21

magnetic resonance angiography

PCV-9, PNE-68

magnetic resonance arthrography

PMS-52, PMS-62

magnetic resonance enterography

PGI-58, PGI-62, PGI-63, EM-8

magnetic resonance entrography

PGI-51, PGI-62, GI-5

magnetic resonance imaging PMI-2,

MS1-3, PNE-3, PNE-4, NE1-3,

PMS-3, PMS-4, CV-2, PGI-3,

FN-R2, EM-R2, PGI-70, PGU-7,

PFN-3, EM-5, PGI-4, PMS-12,

PNE-11, FN-12, ON-1, PFN-4,

PMS-15, PNE-13, NE1-5, FU-4,

PON-15, PFN-5, NE2-4, PMS-18,

ON-2, CV-8, MI-3, PMS-22, PMI-14,

NE1-R2, PMI-15, PCV-4, PNE-21,

PFN-6, PNE-23, NE1-1, PON-21,

PNE-25, EM-9, MS2-9, MS1-1,

PNE-28, PMS-37, PMS-41, PGI-35,

PNE-32, PCV-7, PFN-11, PNE-35,

PNE-37, PMI-25, PGI-43, PNE-39,

PMS-44, PNE-45, PNE-47, PON-31,

PNE-48, PNE-51, PMS-52, PNE-54,

PNE-55, PFN-19, PNE-58, PNE-59,

PMS-56, PON-36, PON-37, PON-38,

PMI-31, PMI-32, PMS-61, PNE-63,

PNE-64, CV-1, CH-11, MS2-8,

PGU-38, CV-10, EM-11, FN-1,

PNE-67, PNE-68, GI-12, NE1-13,

EM-R1, PMS-66, CV-6, FN-4,

MS2-R1, FN-5, FU-5, EM-13,

NE1-2, GI-13, GI-4, CV-11, GI-17,

GI-14, NE1-14

magnetic resonance imaging

morphometry NE2-1

magnetic resonance imaging-positron emission tomography MI-3

magnetic resonance imaging, advanced

applications NE1-2

magnetic resonance imaging, arterial

spin labelling PNE-11, NE1-12,

PNE-20

magnetic resonance imaging, brain, fast

magnetic resonance imaging, cardiac

CV-4, CV-5, PCV-2

magnetic resonance imaging, cine FN-4

magnetic resonance imaging, diffusionweighted FN-2, ON-5, EM-9, PGI-51, PNE-65, NE1-13, EM-8, EM-10

magnetic resonance imaging, dynamic contrast-enhanced PNE-56, PGI-51

magnetic resonance imaging, foetal

PFN-2, PFN-7, PFN-8, PFN-12,

PON-34, EM-4, PFN-24

magnetic resonance imaging, foetal

bowel PFN-15

magnetic resonance imaging,

high-field PNE-20, PNE-24

magnetic resonance imaging, low field

EM-14

magnetic resonance imaging, pelvis

PGU-26, PGU-39

magnetic resonance imaging,

perfusion MS2-5

magnetic resonance imaging,

susceptibility-weighted PNE-6,

PNE-66

magnetic resonance imaging,

volumetric PNE-50

magnetic resonance imaging,

whole-body MS2-R2, ON-6

magnetic resonance spectroscopy

NE1-3, PNE-47

magnetic resonance urography

PGU-12, PGU-14, GU-2, GU-5

magnetoencephalography PNE-55,

PNE-63

malformation PNE-37, PCV-8, PNE-45

malformation, arteriovenous, brain IR-1

malformation, central nervous system,

congenital PNE-44

malformation, cystic adenomatoid,

congenital $\mathrm{CH}-14$

malformation, ear PNE-10

malformation, kidney GU-1

malformation, lymphatic PFN-10,

PIR-8

malformation, of cortical development
PNE-54

malformation, vascular IR-R2

malformation, venous PMS-5, PIR-4

malformations of cortical development PNE-55

malrotation EM-5, PGI-42, GI-19

management IR-R1

mass lesion PON-13, PON-18,

PMS-32, PGI-39, PGU-28, PFU-3,

PNE-59, PON-38, PNE-65, MS2-14

mass lesion, bone IR-4

mass lesion, brain PNE-12, NE1-1,

NE1-13

mass lesion, chest, benign PCH-19

mass lesion, hepatic, focal GI-11

mass lesion, liver PON-28, PGI-39

mass lesion, neck PON-6, PON-9,

PFN-26

mass lesion, ovary PGU-26

mass lesion, paratesticular PGU-33

mass lesion, pelvis PGU-25

mass lesion, soft tissue PON-25, PMS-56

mass lesion, testicle PGU-31

mastoid PNE-23

maxillary antrum PON-23

Mayer-Rokitansky-Kuster-Hauser

syndrome PGU-39

mediastinal shift PCH-22

mediastinum $\mathrm{CH}-15$

medico-legal PMI-13

megaureter GU-4

melanin PNE-34

meningoencephalitis PNE-15

mental retardation NE1-6

Meso-Rex bypass GI-8

metabolism, inborn error of PNE-26

metaphysis PMS-63

metastasis PON-4

metastasis, drop NE1-13

Mickey Mouse-sign GU-10

microangiopathy NE2-R1

microlithiasis, testicle GU-11

micturating cystourethrography, see

voiding cystourethrography

mid-aortic syndrome PIR-1

midazolam MI-1

middle-ear cavity PON-23

migraine PNE-43

morbidity and mortality PRS-19

morphology MS2-4

morphometry, optimal voxel-based

NE1-6

motor vehicle accident, see road 


\section{traffic accident}

Moyamoya PNE-61

mucormycosis PMI-23

Mullerian duct PGU-16

multi-centre trial ON-4

multi-institution PNE-67

multi-scanner PNE-67

multifocal PGI-35, PMS-42, PGI-39

multimodality PGU-25

multiplanar imaging $\mathrm{CH}-7$

muscle MS1-2, PMS-67

Mycocbacterium tuberculosis $\mathrm{PCH}-4$ myelination, accelerated PNE-5

naegleria PMI-23

nasal bone PNE-33

neck PMI-17

necrosis MI-2

necrotising enterocolitis PFN-1

neonate GU-1, PFN-1, PNE-4, PGU-3, NE1-3, PGI-71, FN-12, FN-10, NE1-5, FN-7, PON-15, PFN-5, PGU-13, PCH-10, PGI-19, PMS-30, PCH-18, PRS-14, PFN-11, PFN-13, PMI-26, PFN-16, PNE-46, PFN-17, PNE-51, PNE-69, PNE-70, EM-12, FN-9, NE1-2, PFN-25

neoplasm PON-15, PON-24, PFU-3, ON-3, IR-4

neoplasm, kidney PON-26

neoplasm, ovary PGU-26

nephroblastoma PGU-23, PON-26, PON-37, ON-8

nephroblastomatosis PON-37

nerve injuries MS1-1

neuro-endocrine cell hyperplasia

PCH-6

neuroblastoma PNE-2, PON-19,

PGU-23, PON-36, PGU-37, ON-R1

neuroblastoma, stage 4S PON-22

neurodevelopmental delay EM-R3

neuroimaging PNE-32, PNE-50

neurometabolic disease PNE-47

neurosurgery PNE-46

non-accidental injury PMS-8,

PMS-13, NA-6, PMS-45, NA-2

non-neoplastic PFN-5

non-sedation PMI-31

normal variant MS1-3, GU-9

nuclear medicine PMI-33, PRS-21

nutcracker PGI-2

obesity PGI-56 obstruction, intestinal PGI-7, PGI-19

obstruction, ureteropelvic junction

IR-3, PGU-12, GU-2, GU-3

obstruction, urinary PFN-3

ocular PNE-58

oculoauriculofrontonasal syndrome

PNE-33

oesophageal atresia PGI-10

oesophagography PGI-8, PCV-7

omphalocele PFN-4

optic nerve-invasion $\mathrm{ON}-1$

orbit PNE-57

ossicles PNE-18

ossification MS1-3, PNE-16

ossification, calcaneum FN-14

osteochondral lesion PMS-64

osteofibrous dysplasia PMS-1

osteogenesis imperfecta PMS-21

osteomyelitis PMS-18, PMI-23,

PMS-42

osteomyelitis, chronic recurrent

multifocal MS2-R2

osteonecrosis PMS-18, MS2-8

osteosarcoma PON-12

out-patient PMI-31

outcome PFN-20, PGU-35, PFN-25

outcome, neurodevelopmental NE2-2

ovarian torsion PGI-48

ovary PGU-9, GU-R2, PGU-39

pain, abdominal PGI-73, PGI-28,

GI-1, PGI-40

pain, abdominal, recurrent GI-3

pain, right lower quadrant GI-2

pancreatitis PGI-22

Panner disease PMS-64

panniculitis, cytophagic histiocytic

PMI-21

panton-valentine leucocidin toxin

MI-2, PMS-19

parallel imaging CV-10

patient safety PGI-30

pectoralis major muscle MS1-2

pectus excavatum $\mathrm{CH}-11$

pelvic floor PGU-32

pelvis, transverse diameter PGU-21

perfusion PFN-17, GI-7, FN-9, EM-13

perichondrium $\mathrm{PFN}-19$

periosteal reaction PMS-13

peritonitis, sclerosing PGU-34

phantom PNE-67

phase contrast CV-1, CV-10, EM-R1

phenotype PNE-33 physical stress CV-5

physician extender PED-4

physician's assistant PED-4

physiology PCH-17

picture archiving and communication

system PRS-8

pig tail PIR-2

pilomatricoma PMS-51

pilomatrixoma PMS-51

pleuropulmonary blastoma $\mathrm{PCH}-8$

pneumatosis intestinalis PGI-40

pneumocystis PMI-23

pneumonectomy syndrome $\mathrm{CH}-15$

pneumonia PCH-1, PCH-14, PMI-23

pneumonia, Pneumocystis jirovecii

PCH-16

pneumonitis, lymphocytic interstitial

PCH-4

Poland syndrome MS1-2

portal vein PGI-34

portal vein thrombosis PIR-5

position, line and tube PMI-20

positron emission tomography NE1-4,

MI-3, PFU-2, PFU-3, PNE-63,

FU-R1, FU-6

positron emission

tomography-computed tomography

FU-3, FU-1, FU-2

positron emission tomography/

computed tomography PFU-1, FU-4,

FU-5

post mortem PMI-1, PMI-26, EM-12

post operative PGI-10

post traumatic PGU-14

post-processing filters PRS-2

post-processing filters, adaptive PRS-1

posterior urethral valves PGU-17

pre-medication MI-1

precocious puberty PGU-5, PGU-20

premature FN-11, PFN-22

prenatal diagnosis FN-R2, FN-R1

preoperative $\mathrm{CH}-7$

prevalence MS2-1

primitive neuro-ectodermal tumour

PON-11, PON-12

procedure, surgical PMS-65

procollagen type III PCV-2

prophylaxis MS2-R1

propranolol ON-9

prospective randomised study PRS-2

prospective study EM-1

protocol PMI-24

pseudoaneurysm PMI-11 
pseudoaneurysm, spleen PGI-5

pseudocyst, cerebrospinal fluid PNE-36

pseudotumour, pancreatic PGI-17

puberty GU-R1

puberty, precocious GU-R1

pulmonary sling PCV-7

pulsatility index GU-R1

purpura fulminans PMS-15

pyeloplasty IR-3

quality PED-2

quality assurance RS-13, PMI-22,

PRS-14, PGI-31, PNE-67

quality improvement PMS-34, PRS-19

rabbit MS1-4

radiation PRS-10, PRS-15, PRS-19,

CV-9, RS-14

radiation dose PRS-1, RS-4, PRS-10,

RS-3, RS-12, CH-12, RS-10,

PRS-21, PRS-22

radiation dose modulation RS-5

radiation dose reduction RS-R1, RS-8

radiation dose, breast $\mathrm{CH}-13$

radiation dose, diagnostic reference

levels PRS-13, RS-1

radiation dose, low $\mathrm{CH}-13$

radiation exposure RS-13, ON-8

radiation exposure, occupational PRS-18

radiation protection PRS-18

radiation safety PRS-6, PRS-10, RS-6,

PRS-14, PRS-17, RS-R2, RS-1,

PRS-21

radiation therapy $\mathrm{ON}-2$

radiation, organ dose RS-3

radiography PGI-6, PMS-21, PMI-20,

PMS-29, PMS-43, PMS-58

radiography, abdomen GI-1

radiography, chest $\mathrm{CH}-9$, RS-13,

PMS-24, PRS-14

radiography, digital PMI-13, RS-13,

PMS-34

radiography, digital, detector PRS-11

radiography, pelvis PMS-25

radiography, post mortem $\mathrm{FN}-14$

radionuclide PNE-52

reconstruction, three-dimensional

PMS-17

reduction PGI-18

reduction, hydrostatic PGI-1

reflux, gastro-oesophageal PGI-33,

PGI-67

reflux, vesicoureteric PGU-8, GU-8,
GU-6

renal cell carcinoma PON-31

renal function PGU-4

renal scarring PGU-10

renal vein $\mathrm{FN}-7$

renal volume PGU-10

report content PGI-31

report structure PGI-31

resistive index PMS-16, NE2-2

respiration $\mathrm{PCH}-17$

respiratory distress syndrome $\mathrm{CH}-2$

respiratory trigger $\mathrm{PCH}-15$

response assessment EM-10, ON-4

response criteria in solid tumours

ON-7

restraint PMI-30, PMI-35

retinoblastoma $\mathrm{ON}-1, \mathrm{ON}-2$

retinoblastoma, trilateral NE2-4

review PON-24, PMI-24, NA-2

rhabdoid/teratoid PON-16

rhabdomyosarcoma PON-12, PON-14,

ON-7, PON-20, PON-23,

PON-33, FU-R1

rhabdomyosarcoma, abdominal

PON-17

rickets PMS-53, NA-4

ring-enhancement PNE-30

risk reduction PRS-15

road traffic accident PMI-35

sacral dimple PNE-70

safety PMI-15, PMI-18, PGI-32

sarcoma, embryonal PON-10, PON-38

sarcoma, epithelioid PON-11

sarcoma, Ewing PON-12

sarcoma, fibro- PON-11

sarcoma, synovial PON-11

sausage

scimitar syndrome PCH-22

scintigraphy, bone PON-19

scintigraphy, HIDA PGI-44

scintigraphy,

metaiodobenzylguanidine PON-19

scintigraphy, renal PGU-10

sclerotherapy PIR-8

scoliosis PMS-17, PMS-34, PNE-62,

PMS-61, PMS-65, GI-7

scoliosis, idiopathic NE2-1

score, abbreviated injury PMI-35

scrotum PGU-3, PGU-28

sedation PMI-2, PMI-14, PMI-15,

PCH-15, PMI-18

sedation, oral PMI-19 seizure PNE-63, NE1-8

semicircular canals NE2-1

shoulder PMS-52, PMS-66

shoulder instability PMS-62

shunt series PNE-9

shunt, pretricuspid PCV-4

shunt, systemic-to-pulmonary PCV-4

shunt, transjugular intrahepatic

portosystemic PIR-5

sialorrhoea PNE-40

sickle cell disease PMS-18, NE1-14

sigmoid colon PGI-6

signal-to-noise PNE-67

skeletal dysplasia PMS-23, PMS-24,

PMS-25, PFN-9

skeletal survey PMS-8, NA-6

skin PON-22

skull base PNE-16

small parts MS2-11

socio-economic status PRS-3

soft tissue sarcoma PON-11

sorbitol PGI-63

specialisation RS-R2

spectrum PGI-10

spherocytosis, hereditary PGI-25

spinal cord PNE-3, PNE-4

spinal cord, focal lesion PNE-48

spine PNE-35, PNE-53, PMS-60,

PNE-65, RS-12

spirometry $\mathrm{CH}-10$

spleen PGI-70, PGU-7

spleen, wandering PGI-52

stacking $\mathrm{CH}-15$

staff PMI-8

Staphylococcus aureus MI-2, PMS-19

Staphylococcus aureus, meticillin

resistant PMI-23

steady-state free precession PCV-9

stem cell PCH-4

stomach, anatomy PGI-33

stone PGU-1

stricture PGI-68

stricture, oesophageal

stroke PNE-27, PNE-28, PNE-62,

PFN-25

subscapularis muscle PMS-66

superior mesenteric artery GI-15, PGI-2

suprarenal mass ON-R1

surgery PNE-23, GI-7

surgery, craniovertebral PNE-36

synchrotron PMS-33

syndrome PMI-5, PNE-37,

PMS-55 
T2* GI-14

tachypnoea $\mathrm{PCH}-18$

technique PGU-15

teratoma PFN-26

teratoma, foetal intrapericardial PON-34 teratoma, foetal mediastinal PON-34 testicle PGU-7, PGU-29, GU-11 tetralogy of Fallot CV-5, PCV-2 thalamus, bilateral PNE-32 thelarche PGU-20 therapy, enzyme replacement thermal imaging MS1-6 thorax, see chest three-dimensional PCH-17, PMS-44, CH-7, RS-12, EM-R1

thrombolysis PIR-5

thrombosis FN-10

thrombosis, cerebral sinus vein FN-12 thrombosis, portal vein GI-8 thymus, rebound hyperplasia FU-2 thyroid IR-5

tibia MS1-4

tolerance PGI-63

torsion PGU-28

torsion, omental PGI-29, PGI-52

torsion, spermatic cord PGU-18

torsion, testicular PGU-18, PGU-29

torsion, testicular appendages GU-10

torticollis PMS-54

total anomalous venous return PCV-1

trachea $\mathrm{PCH}-13$

tracheobronchomalacia $\mathrm{CH}-8$

tractography NE1-9, NE1-8

transplant, bone marrow PCH-4, MS2-8

transplant, intestine PGI-57

transplant, liver PGI-9, PGI-20

transplant, lung $\mathrm{CH}-10$

transplant, solid organ $\mathrm{PCH}-4$

transposition of the great arteries FN-13

trauma, see injury

treatment MS2-3

tube, gastrojejunal feeding PGI-67

tube, indwelling PMI-20

tube, nasogastric PGI-30, PGI-31,

PGI-32

tuberculosis CH-15, PMS-22

tuberculosis, extrapulmonary PMI-10

tuberculosis, neuro PNE-25

tuberous sclerosis PMI-24, PMI-32

tumour, see mass lesion

tumour, germ cell PGU-31

tumour, lipomatous PMS-7

tumour, myofibroblastic PCH-19 tumour, second primary $\mathrm{ON}-2$

tumour, vascular PGI-14

twins, conjoined EM-R2

ulcerative colitis PGI-3

ultrasound PGU-1, PGU-2, PGI-1, GU-1, GI-3, PFN-1, FN-11, PGU-3, PGU-4, PGU-6, PGU-7, PGI-72, GU-8, GI-6, GI-10, CH-3, ON-R2, PGU-9, EM-3, PGI-9, PGI-11, ON-9, PGI-13, PMS-16, PON-15, PFN-5, PGI-16, PGU-11, GI-9, PMI-7, PMS-20, MS2-4, PON-18, PGU-13, PGU-14, PCH-10, PMI-16, PNE-22, EM-2, PGI-25, MS1-1, PMS-32, PGI-27, PNE-26, MS1-2, PGI-28, GI-1, PCH-19, PGU-24, PGI-35, PFN-11, PGU-28, PGU-29, PNE-37, PFN-13, PGI-40, PGI-42, PNE-39, PGI-44, PGI-45, PFN-16, PGU-31, PGI-48, PNE-58, PNE-59, PGU-33, PMI-33, PFN-20, PGU-35, EM-6, PGU-38, GU-R2, PNE-69, PNE-70, PGI-61, MS2-11, GI-19, GI-5, PMS-67, GI-2

ultrasound screening MS2-2 ultrasound, chest PCH-14, CH-6 ultrasound, contrast-enhanced GU-9, GI-11, EM-7

ultrasound, high-resolution $\mathrm{PON}-25$ ultrasound, lung $\mathrm{CH}-2, \mathrm{CH}-4, \mathrm{CH}-6$ ultrasound, neuro NE2-2, PFN-23 ultrasound, pelvis PGU-26 ultrasound, quantitative EM-1, MS1-4 ultrasound, renal PGU-10 ultrasound, transfontanellar PNE-45, PNE-46

ureter, ectopic PGU-19

ureterocele PGU-35

ureteroscopy RS-14

urethra GU-9

urinary GU-4

urinary tract GU-3

urinary tract, lower PFN-3

urinoma PGU-14

urology PGU-6

uterus didelphys GU-1

uterus, hemi- PGU-39

utilisation RS-R2, RS-R3

vaccination, rota virus PGI-27

vaginoscopy PGU-19

varicocele PGU-2 vascular PCV-9

vascular anomaly PMI-5, PGI-14, PNE-56, IR-R2

vascular ring $\mathrm{PCV}-7$

vasculitis

vasculitis, central nervous system PNE-68

velocity-encoded imaging $\mathrm{CV}-4$

ventilation/perfusion $\mathrm{PCH}-21$

ventricle size PFN-23

ventricle, right $\mathrm{CV}-8$

ventricle, single CV-6

vertebra PMS-60

vessel, tortuous PNE-62

virtual imaging PGU-19

vitamin D PMS-53

voiding cystourethrography PGU-8, PGU-15, GU-6, PGU-17, PGU-24, PGU-36, PED-4

voiding uroultrasound GU-7

volume EM-R1

vomiting GI-15, PGI-2

water-bath MS2-11

white matter NE1-3, NE1-5

Wilms' tumour, see nephroblastoma

Wilson disease PGI-13

workforce PMI-8

wrist PMS-3, PMS-4, MS2-9

\section{Index of abstract authors}

Abbara, Suhny CH-13

Abbas, Syed Zafar PGU-15, PMS-23

Abd Manaf, Zaleha PCH-10

Abdalla, Wael PGI-64, PGI-65

Abdar Esfahani, Shadi PGU-19, PON-23

Abdeen, Nishard PNE-35, PON-33

Abdul Hamid, Hamzaini GI-2

Abdullah, Asif PNE-39, PON-29

Abernethy, Laurence NE1-1

Abramson, Sara ON-11, PON-36, PON-38

Abulaban, Osama GU-6

Adeb, Melkamu GI-5

Adeyiga, Adebunmi PED-2, PGI-50

Adikibi, Boma IR-3

Adzick, Scott N FN-5

Agarwal, Prachi PCV-7

Aguilera, Dolly NE1-13

Ahmad Sarji, Sazilah PGU-10

Ahmad, Tahani PRS-19 
Ahmed, Aadil PMI-3

Ahuja, Chirag CH-3

Aida, Noriko PNE-10

Akay, Sinan PGU-4

Akgun, Veysel PGU-4

Akhtar, Sameen PGU-21

Aksoy, Murat NE1-R1, PNE-65

Al Gathradi, Mohamed PON-33

Al-Hashimi, Hakima PCH-8, PCH-18, PNE-17, PON-14

Alagappan, Vijay EM-11

Alam, Mostyn PGU-6

Alavi, Afshin MI-2, PMI-9, PMI-10,

PMS-18, PMS-19, PNE-28

Alazraki, Adina PON-35

Alberti, Corinne FN-2

Alcala-Galiano, Andrea PGU-18

Alconcher, Laura PFN-21, PGU-35

Alexander, Alan PNE-38

Ali, Kamran EM-12, MS2-10, PMI-26

Alison, Marianne FN-2, PMS-17

Alizai, Naved GI-9

Allan, Rosemary PGU-17

Allbery, Sandra EM-6

Allen, Pamela PGI-22

Alley, Marcus CV-10, EM-11, EM-R1, PMS-44

Alli, Kasumawati PGU-10

Allison, Travis PNE-70

Alomari, Ahmad IR-R2, MS2-14

Alsip, Chris CH-12, RS-8

Altinok, Deniz PNE-34

Álvarez-Muelas, Alberto PCV-3

Alvarez, Marina EM-9

Amaral, Joao IR-2, PMS-5

Amarnath, Jaya PGI-25, PMS-24, PRS-13

Ambrosetti, Laurent PRS-6

Amici, Lucia PFN-3, PGI-73, PGI-70, PON-22

Amin, Raouf EM-13

Amirabadi, Afsaneh FU-5, MS1-4

Ancliff, Philip PGI-25

Anderson-Evans, Colin CH-12, RS-8

Anderson, Helen PMS-13

Anderson, Joanna PGU-17

Andersson, Henrik PRS-1, PRS-2

Andre, Jalal NE1-R1

Andrew, Ramsden $\mathrm{CH}-8$

Andronikou, Savvas CH-15

Angel, Erin CH-12, RS-8

Annam, Aparna PGU-33

Anoni, Clara PNE-64
Anooshiravani, Mehrak EM-3, PRS-6

Anosike, Chinedum PMI-4

Anthony, Evelyn PFN-14, PGU-26,

PMI-35, PON-26

Anton, Christopher PMS-46

Antonello, Monica PFN-8

Anupindi, Sudha GI-5

Apgar, Bruce PRS-14

Apsey, John PGI-4, PMI-20

Arevalo, Juliio PGI-27

Arevalo, Julio CH-6

Armspach, Jean-Paul PFN-6

Armstrong, Euan PGI-4

Arnold, Ryan IR-R2

Aroca, Ángel PCV-3, PCV-4

Arora, Gaurav GI-6

Arslanoglu, Atilla PNE-13

Arthurs, Owen EM-5, GI-15, PCH-3,

PGI-2, PMI-2, PMS-6, PMS-8,

PMS-9, PNE-3, PNE-4, PON-8

Arvaniti, Mairoula PMI-7, PNE-16

Asamoto, Jane PRS-17, PRS-22

Asghar, Sheila PNE-43

Aspirot, Ann PFN-16

Attard, Alexia PMS-14

Attili, Anil PCV-7

au-yong, kong jung PMS-26

Austin, Joan PGI-30

Avcin, Tadej PIR-1

Avenarius, Derk F M PMI-14

Averill, Lauren PGI-56

Avni, Fred FN-7

Avula, Shiravam PNE-23

Avula, Shivaram NE1-1, PMI-4, PNE-5

Axelson, Rick PED-3

Aziza, Albert PGI-63

Azzie, Georges GI-2

Baban, Anwar MS1-2

Babyn, Paul MS2-R1, PGI-63,

PMS-47, PRS-14

Badosa, Joaquim PCH-11, PNE-14, PNE-18, PON-21

Badve, Chaitra PNE-41

Bagga, Dr Deepak PGU-14

Bagiazidou, Eleni PCH-14

Bailey, Smita PMS-52

Bailey, Zachary PNE-49

Bainbridge, Alan FU-4, ON-5

Bainbridge, Jonathan PFN-5, PON-15

Bakalinova, Daniela PNE-29

Baker, Kenneth MS1-6

Ballah, Deddeh PIR-8
Ballesteros, Martha PMS-60

Bammer, Roland NE1-R1, PNE-65, PNE-66

Bar Oz, Benjamin FN-10

Baran, Galina PMS-60

Barbarisi, Julia PMS-53

Barber, Ignasi EM-9, PGI-24, PGI-26

Bardeggia, Laura PMI-21

Bardo, Dianna PCV-8, PRS-17, PRS-22

Barelli, A RS-8

Bareño, Sandra PGI-52

Barnacle, Alex IR-2

Barnes, Craig PMS-52

Barnes, Patrick PFN-28, NE1-R1,

PNE-65, PNE-66

Barnewolt, Carol FN-4, GU-R2, PGU-39

Barnhart, Douglas PGI-67

Barreto, Mithya CH-13

Barrett, Sarah PMI-18

Barsness, Katherine GI-19

Barth, Richard PFN-28, PFN-15

Bartlett, Murray PMS-3, PMS-4

Barton, Katherine PCV-8

Bartz, Brett RS-7

Baruah, Barindra Prasad PCH-7, PGU-15, PMI-13, PMS-22, PMS-23

Baruah, Bp PMS-16

Baruah, Dhiraj PMS-61

Baskin, Jr., Henry J. PMS-46

Batista, Edward ON-3

Battelino, Nina GU-7

Bean, Adrienne PGI-57

Beaule, Paul MS2-7

Bebbington, Michael FN-5

Beer, Meinrad EM-1, EM-14

Beiderman, Jessica NE2-2

Bekesinska-Figatowska, Monika ON-9

Bekiesinska-Figatowska, Monika

PNE-19

Belarbi, Nadia FN-2

Belev, George PMS-33

Bellah, Richard PGU-37

Ben Ely, Anna FN-9, GU-10, PFN-17

Ben-Haim, Simona FU-4

Ben-Shlush, Aviva FN-11

Bendavid, Guila PRS-19

Benders, M. FN-12

Beneck, Debra PGI-59

Benson, Jane PGI-47

Berden, Pavel PIR-1

Bermudez, Dustin CH-14

Bernard, Chantal PFN-17 
Bernardi, Bruno NE1-9, PNE-25

Bertocchini, Arianna GI- 8

Bethapudi, Sarath PGI-18

Bhatia, Anmol PGI-15

Bhutta, Sadaf CV-9

Biko, David GI-5

Bilaniuk, Larissa T. EM-4, PNE-53

Biller, Jeffrey GI-4

Billette de Villemeur, Thierry FN-R2

Billups, Catherine FU-R1

Bilska, Katarzyna ON-9

Binkovitz, Larry PMS-45

Biondetti, Pietro PGI-71

Bird, Helen PMI-12, PNE-9

Birkemeier, Krista CH-11

Bisset, III, George PCV-9

Bitters, Constance PFN-24

Bixby, Sarah MS2-12, NA-R1

Blanch, Jose PNE-14, PNE-18

Blanch, Josep PCH-11, PON-21

Blanchette, Victor MS2-R1

Blaser, Susan CV-1

Blondiaux, Eléonore FN-R2

Blumfield, Einat PFN-22, PGU-24

Boavida, Peter MS1-5

Boddaert, Nathalie NE1-12

Boechat, Ines PGI-57

Boiselle, Phillip CH-7

Bojic, Zoran PRS-19

Bokhari, Syed MS1-6

Bolli, Valeria PFN-3, PGI-73, PGI-70,

PMI-21, PON-6

Bonekamp, David PNE-56

Borg, Philip PGI-3, PMI-11, PNE-8

Borzani, Irene PGI-71

Bosemani, Thangamadhan PGI-47, PNE-48

Boylan, Emma GI-19, PGI-68

Boysen, Dennis RS-6

Bracken, Jenny PMI-19, PMS-2, PMS-3, PMS-4

Brady, Samuel ON-8

Bragoszewska, Hanna ON-9, PNE-19

Braithwaite, Kiery PON-35

Brandão, Júlio PGU-27

Brandao, Leonardo PMS-5

Brau, Anja EM-11

Bret-Zurita, Montserrat PCV-3, PCV-4

Bret, Monserrat PGI-14, PMI-5

Brill, Paula PGI-59

Brisse, Hervé NE2-4, ON-2

Britton, Lunetha MS2-8

Brock, John GU-4
Brody, Alan CH-12, RS-8

Brown, Robert Clark PNE-71

Brown, Stephen FN-R1

Brown, Yolanda PFN-22

Browne, Lorna PGU-33

Brunelle, Francis NE1-12

Bryce, Thomas PON-10

Brzewski, Michal PGI-16

Buchmiller, Terry FN-R1, PFN-10

Bueno, Javier PGI-24, PGI-26

Bulas, Dorothy NA-2, PNE-52,

PNE-61

Buonomo, Carlo NA-R1

Burak, Jarett PON-27

Burke, Derek MS1-R1

Bush, Patricia ON-3

Byrne, Angela PNE-51

Cacaci, Silvia PCH-4

Cadavid, Lina EM-9, PGI-24, PGI-26

Cahill, Anne Marie PIR-8

Cain, Timothy MI-3

Calder, Alistair PCH-4, PCH-5,

PMS-24, PMS-25

Calder, Alister CH-10

Calderon, Paul EM-11

Calevo, Maria Grazia MS1-1, MS1-2

Callahan, Michael MS2-11, PMS-67,

RS-1

Calvo-Garcia, Maria PFN-26, PON-34

Calvo-Garcia, Maria A. PFN-24

Campbell, Naomi PMI-18

Canazza, Lorena PGI-71

Cannatà, Vittorio NE1-9

Cariati, Ettore PFN-7, PFN-8

Carmichael, Jim CH-10

Carmignani, Marcia PNE-45, PNE-46

Carnevali, Alessandra PGI-71

Carney, Olivia PMS-29

Carpineta, Lucia FN-1

Carre, Matt NA-3

Carrino, John A PNE-56

Carroll, Daniel PGU-6, PGU-7

Carsen, Sasha MS2-7

Caruso, Paul PMI-24

Caserta, Melanie PGU-26

Cassady, Christopher FN-1

Cassia, Guilherme FN-9, GU-10,

NE2-3, PFN-17

Castaño, Marta PGU-18

Castelijns, Jonas A. NE2-4, ON-2

Castellote, Amparo EM-9, PGI-26

Castile, Robert $\mathrm{CH}-5$
Castro-Aragon, Ilse PGU-28, PGU-29

Castro, Denise PMS-51

Catanzano, Tara PGI-37, PGI-38,

PGI-46, PGU-30, PMS-35, PNE-31

Catena, Nunzio MS1-1

Caur, Vandan PON-36

Cerwinka, Wolfgang GU-2

Chakraborty, Subhasis PRS-13

Chalard, François FN-R2

Chan, Frandics CV-R1, EM-R1, RS-10

Chan, Godfrey Chi-Fung PNE-6

Chan, Mei Yoke PON-14

Chan, Verena Sau Wai PMI-15

Chang, Tou En Kenneth PON-4

Chang, Yoon Sil NE1-5

Chapman, Dean PMS-33

Charsoula, Anna PMI-7

Chatterjee, Somenath PGI-7

Chaturvedi, Abhishek PNE-32

Chaturvedi, Apeksha PNE-32

Chaudhary, Kiran PMS-16

Chaudry, Gulraiz MS2-14, PFN-10

Chauvin, Nancy PMS-59

Chaves, Ian EM-13

Chavhan, Govind PIR-7, PNE-42

Che, Li CH-R3

Chen, Lu ON-4

Chen, Michael PGU-26, PON-26

Chen, Yi-Fang PON-9

Cheng, Alven PGI-48

Cheng, Jack CY NE2-1

Cheon, Jung-Eun FN-8, MS2-5

Chetty, Shilpa PFN-15

Cheung, Eddie CV-8

Cheung, King Kenneth FU-4

Cheung, Pt PMS-21

Cheung, Yf CV-8

Chew, Felix PMS-36, PMS-37,

PMS-38, PMS-39

Chhadia, Sumit PGU-40

Childs, John PNE-38

Chiong, Brian PGU-24

Chippington, Sam IR-R1

Cho, Steven ON-4

Cho, Young ah PCH-9

Choi-Rosen, Jeane PON-27

Choi, Hye Young PGU-9

Chong, Kling PNE-3, PNE-4

Chou, Pauline IR-5

Chow, Jeanne GU-3, GU-5, RS-14

Christos, Paul PGI-59

Chrysanthopoulou, Athina PGI-23

Chu, Winnie CW GI-3, NE2-1 
Ciccarone, Antonio PNE-20, PNE-21,

PRS-12, RS-5

Clarke, Jonathan PGU-6

Clarke, Maxine NE2-2, PFN-23

Claros, Pedro PNE-14, PNE-18

Coca, David CH-6

Cohen, Mervyn PGI-30, PGI-31, PGI-32, PGI-33, PGU-23, PMI-22, PMI-36, PMS-34, PRS-14

Cohn, Richard PGU-40

Coley, Brian PNE-69, PNE-70

Coley, Ian PNE-69, PNE-70

Collins, Lee PON-27

Conceição e Silva, João Paulo

PON-11, PON-12

Connolly, Bairbre PRS-18

Connolly, Susan FN-4, PGU-39

Constine, Louis ON-4

Conway, Deborah PNE-40

Cook, P. Christopher GI-7

Coombs, Laura RS-1

Cooney, Joseph MS1-5

Cooper, Julie PMS-32

Cooper, Matthew GU-11

Corno, Lucie EM-2

Corsello, Giovanni PRS-5

Coulon, Philippe RS-5

Courtier, Jesse PGI-62

Craft, Alan PRS-3

Crapp, Seth GU-4, PMS-55

Crossett, Marcus CH-8

Cuesta-López, Emilio PCV-3, PCV-4

Cunningham, Michael PNE-33

Curran, John FU-6

Czerwinska, Katarzyna PGI-16

D’Ambrosio, Giuseppe GI-8

Dagia, Charuta NE1-R2

Daldrup-Link, Heike FU-2, PGI-62

Damilakis, John RS-9

Damphousse, Amelie PGU-31, PON-37

Daneman, Alan GI-2, ON-R1, PFN-18

Danielle, Smyth PMI-18

Daniels, Barret NE1-2

Danin, Joanna MI-2, PMI-9, PMI-10,

PMS-18, PMS-19, PNE-28

Dantuluri, Narasimharaju GI-9

Danzer, Enrico FN-5

Dardanelli, Esteban PMI-32

Darge, Kassa GI-5, GU-8, RS-1

Dargel, Susanne PMS-31

Das, Ashim GI-10

Das, Raj NA-6
Davila, Jorge EM-8, PGI-51

Daw, Najat ON-8

Daw, Stephen MS1-5, ON-5

de Benedictis, Fernando Maria PMI-21

De Cobelli, Francesco GU-R1

de Graaf, Pim NE2-4, ON-2

De Iorgi, Vincenza GU-R1

De La Fuente, Josu PMS-18

de Lange, Charlotte NE1-4

De Ville de Goyet, Jean GI-8

de vivo, Manuela PMI-21, PON-22

De Vries, L. FN-12

De, Prasanjit PGU-15, PMS-23

De, Sujata PNE-23

Decarie, Jean Claude FN-6

deFreitas, R. Andrew CV-3

Deganello, Annamaria GI-11, PGI-5

Deguer, Carlos PFN-20

Deitch, Sarah IR-5, PIR-5

del Pozo Garcia, Gloria CH-6, GI-1,

PGI-27, PGU-18

Delalande, Olivier NE1-9

Delaney, Lisa PMI-33

DeLappe, Eithne ON-11

Delgado, Ignacio PNE-11

Demers, Christine MS2-R1

Dempsey-Robertson, Molly ON-3

Deng, Jie GI-12

Dermentzoglou, Vasiliki PCH-14,

PGU-11

Dery, Julie FN-6

Desilet-Dobbs, Debbie EM-12,

MS2-10, PMI-26

Dev, BHAWNA PGI-1

deVeber, Gabrielle NE1-14

Devito, Rita PNE-25

Dezateux, Carol MS2-2, MS2-6

Dhingra, Smita PMS-22

Dhouib, Amira FN-R2

Di Maurizio, Marco PFN-7, PRS-12

di rocco, Federico NE1-12

Diefenthal, Helmut PMI-34

Dietemann, Jean-Louis PFN-6

Dietrich, Jennifer PGU-38

Dietz, Charles PGI-53, PGI-69, PMI-34

Dietz, Kelly PFN-27, PGI-53, PMI-34

Dillman, Jonathan PCV-7, PGI-35, PMS-43

Dillman, Jonathan R. PMS-41

Dillman, Jonathan R. PON-31

Dinan, David PCH-22

Ditchfield, Michael CH-8, MS1-3,

NE1-R2, PMS-2
DiVasta, Amy GU-R2

Djordjevic, Miroslav PGU-12

Djuricic, Goran PGI-19, PGI-28

Dlangamandla, Salome PON-16

Do, R K PON-38

Dobbs, Nathan EM-12, PMI-26

Doja, Asif PNE-68

Dolia, Elena NE1-3

Don, Steven RS-R3

Donald, Geoff MS2-9

Donaldson, James GI-12, GI-19, IR-5, PGU-40, PIR-5

Donelan, Karen FN-R1

Donnelly, Lane NE1-2, PMI-27,

PMI-28, PMI-27

Donoghue, Veronica PMI-19, PMS-29

Dorfman, Adam PCV-7

Doria, Andrea FU-5, MS1-4, MS2-R1, PMS-47

Doris, Zebedin RS-R1

Dorn, Curt EM-12, PMI-26

Dorr, Katrin PCV-5, RS-R1

Doudounakis, Stavros PGU-11

Doull, Iolo PIR-2

Douvlou, Evanthia PGU-22, PON-25

Dow, David CH-12

Downey, Joe PGI-67

Downey, Ryan T. PON-31

Drubach, Laura PFU-3, PRS-21

Dubois, Josée FN-6, PFN-16

Ducou le Pointe, Hubert FN-R2

Duczkowska, Agnieszka PNE-19

Duczkowski, Marek PNE-19

Duhn, Ryan NA-5, PGU-23

Duigenan, Shauna PGI-39, PMI-24,

RS-11

Duma, Stefan PFN-14

Dumoulin, Charles NE1-2

Dunlap, Hal MS2-7

Duran Feliubadaló, Carmina GU-9

Dutenhefner, Eliane Eliza PFN-13, PGI-40, PNE-37, PNE-45

Dux, Anthony PCH-12, PMI-16

Dydinski, Philip PCV-9

Dydynski, Philip PGI-45

Eastwood, Deborah MS2-6

Eastwood, Deborah M. MS2-2

Ecker, Jeffrey FN-R1

Edelbauer, Monika PNE-22

Edelman, Moris PON-27

Edwards, Andrea EM-5, PMI-2

Edwards, Martin PCH-1 
Egelhoff, John CH-12, RS-8

Ehrlich, Peter PON-31

El-Hawary, Ron GI-7, PMS-65

El-Sayed, Yasser PFN-15

Ellett, Marsha PGI-30, PGI-31, PGI-32, PGI-33

Elliott, Martin PCH-13

Elmaleh-Berges, Monique FN-2

Elsamaloty, Haitham PNE-39

Emans, S. Jean GU-R2

Emerson, Nick NA-3

Engesæter, Ingvild MS2-1

Engesæter, Ingvild Ø. MS2-2

Engesæter, Ingvild Øvstebø MS2-6

Engesæter, Lars B MS2-1

Engesæter, Lars B. MS2-2

Engesæter, Lars Birger MS2-6

Enriquez, Goya EM-9, PGI-24,

PGI-26, PNE-11

Eo, Hong NE1-5, ON-1, RS-4

Epelman, Monica CH-14, PFN-18

Ergaz Shaltiel, Zivanit FN-10

Erlichman, David PFN-22

Espinoza, Anibal PGI-52

Esposito, Marco RS-5

Estroff, Judy FN-1, FN-4, PFN-10

Eugster, Erica PMI-33

Evans, Alison NA-1, NA-R2, PCH-1,

PFN-5, PIR-2, PON-15

Evans, Kelly PNE-33

Fabre, Monique EM-2

Fabrizzi, Giancarlo PFN-3, PGI-73,

PGI-70, PMI-21, PON-6, PON-7,

PON-22

Faerber, Eric PMS-54, PNE-30

Fahey, Frederic PRS-21

Fahmy, Ahmed PGU-1, PGU-2

Faingold, Ricardo FN-9, GU-10, NE2-3, PFN-17, PFN-18

Fanou, Evgenia PNE-29

Fariello, Giuseppe PNE-25

Farmer, Doug PGI-57

Farooq, Ammad PMS-27

Felice, Marc RS-12

Feola, Peter PGI-67

Ferguson, Mark PNE-32

Ferguson, Polly PMS-42

Fernanda Cervantes, Luisa PMS-60

Fernandez, Ramon PFN-1

Ferrari, Celia PGU-34, PGU-36

Ferrario, Matilde GU-R1

Feygin, Tamara EM-4, PNE-53
Filiatrault, Denis FN-6

Findlay, Jennifer PMS-14

Finkelstein, Mark PGI-56

Fischer, Lutz PGI-20

Fishbein, Mark GI-12

Fishman, Steven MS2-14

Fitzgerald, Tj ON-4

Flake, Alan W FN-5

Flamini, Robert NE1-8

Flanagan, Siobhan PGI-54, PGI-69, PMI-34

Flavin, Michael NE2-2, PFN-23

Fleck, Robert EM-13

Fogel, Mark CV-6

Föhr, Anna PCH-6

Fonda, Claudio PFN-7, PFN-8, PNE-20, PNE-21, PRS-12, RS-5

Forbes, Peter NA-R1

Forman, Howard RS-R2

Foster, Judith PGI-4, PMI-20

Franchi-Abella, Stephanie EM-2

Francisco Neto, Miguel Jose PFN-13,

PGI-40, PNE-37, PNE-45, PNE-46

François, Rousseau PFN-6

Frawley, Kieran MS2-9

Freiman, Moti EM-10

Friedman, Seth GI-14

Frush, Donald CH-12, RS-1, RS-7,

RS-8

Fuentealba, Isabel PGI-52

Fujino, Akihiro PIR-3

Fujita, Kazutoshi PCH-15, PNE-10

Funari, Marcelo Buarque de Gusmao

PFN-13, PGI-40, PNE-37, PNE-45, PNE-46

Fusco, Lucia NE1-9

Gabor, Flaviu PMS-17

Gahunia, Harpal FU-5

Gaines, Barbara PGI-64, PGI-65

Galeazzi, Vittoria PFN-3, PON-6, PON-22

Galina, Paraskevi PCH-14, PGU-11

Gallagher, Lora PNE-67

Gallego, Carmen CH-6, PGI-27, PGU-18

Galluzzi, Paolo NE2-4, ON-2

Gan, Bin Kee PMS-11

Ganguly, Arundhuti RS-10

Ganschow, Rainer PGI-20

Garcia Peña, Pilar PGI-24, PGI-26

Garcia, ROSEMEIRE F PGU-27

Garel, Catherine FN-R2

Garel, Laurent FN-6, PFN-16
Gasparini, Flavia Faganello PFN-13, PGI-40, PNE-37, PNE-45

Gasser, Bernard PFN-6

Gassner, Ingmar GU-1, PNE-22

Gattens, Michael PMS-9

Gatti, Marcela PGU-34, PGU-36

Gawande, Rakhee FU-2

Gearhart, John P. PGU-32

Gee, Michael GI-4, GI-17

Geley, Theresa GU-1

Gelfand, Michael FU-1, FU-3

Geller, James PON-28

Gennari, Fabrizio GI-8

Genovese, Elisabetta NE1-9

Gerts, Volker PMS-33

Gervais, Debra RS-11

Gervais, Trevor MS2-9

Ghai, Babita MI-1

Giaquinto, Randy NE1-2

Gibbs, Sally MS1-R1

Gibson, Craig PIR-4

Gie, Robert P CH-15

Gilbertsen-Dahdal, Dorothy PMI-30

Gill, Kara PNE-62

Ginsberg, Jill PGU-37

Giordano, Jessica PRS-4, PRS-5

Githu, Tangayi PMS-60

Glasier, Charles PNE-43

Gold, Garry PMS-44

Goldner, Dana GI-17

Gomez Herrera, Juan Jesus PGI-27

Gomez, Anna PCH-3, PMS-8

Gonzales, Emmanuel EM-2

Gonzalez de Orbe, Gabino CH-6, GI-1, PGI-27

González, Ana-Elvira PCV-4

Goodfellow, Tom GU-6, PGU-20

Goodman, Thomas MS1-6

Goodwin, Susie PGI-18

Gordon, Christopher MS1-4, PRS-18

Gordon, Lena PRS-1, PRS-2

Gorelik, Natalia PFN-18

Göricke, Sophia NE2-4, ON-2

Goske, Marilyn RS-1, RS-R2

Goussard, Pierre CH-15

Grafendorfer, Thomas EM-11

Grandage, Victoria PON-5

Grant, Frederick GU-5, PRS-21

Grasparil, Angelo Don PGI-62

Grattan-Smith, Damien GI-13,

GU-2, NE1-8

Grattan-Smith, Damien J NE1-13

Graves, Martin EM-5 
Greenberg, S Bruce CH-R2, CV-9

Greer, Mary-Louise MS2-9,

PGI-63, PMI-8

Grevent, David NE1-12

Grignon, Andrée FN-6, PFN-16

Grimaldi, Chiara GI-8

Groenendaal, F. FN-12

Grosse-Wortmann, Lars CV-1

Grover, dr shabnam bhandari PGU-14

Groves, Ashley FU-4

Gruss, Joseph PNE-33

Grynpas, Marc MS1-4

Grynspan, David EM-8

Guandalini, Michael MS2-9

Guarnieri, Yanina PGU-36

Guillerman, Robert CH-1

Guimaraes, Carolina PFN-26, PON-34

Guimaraes, Carolina V. A. PFN-24

Guleria, Saurabh PMS-61, PNE-60,

PNE-62

Gulino, Pietro PRS-12

Gunny, Roxanne PNE-3, PNE-4

Gupta, Neil GU-6

Gutierrez-Larraya, Federico PCV-3

Gutierrez, Julia PGI-13

Gwal, Kriti PMS-49, PMS-53

Ha, Sau-Yin PNE-6

Habenicht, Rolf PMS-30

Haber, Marc PGI-46

Hadzic, Dino GI-11

Hager, Lacy PMI-31

Hagisawa, Susumu PNE-2

Hahn, Jin PFN-28

Hain, Sharon FU-4

Hall, Toni IR-R1

Halpern, Elkan FN-R1

Halsey, Michael PFN-14

Hamada, Khaled PMS-63

Hanley, Frank CV-R1

Hanquinet, Sylviane EM-3, PRS-6

Haque, Saira PCH-5, PCH-13

Hara, Hiroko PCH-16

Hara, Masaki PFN-4

Harave, Srikrishna PGI-11, PMS-27, PON-13

Hargreaves, Brian EM-11

Harisinghani, Mukesh GI-4

Harris, Matthew CV-6

Harrison, Sara NA-1, NA-R2, PCH-1

Hassona, Mohamed PGU-1

Hater, Dianne PMI-27, PMI-28, PMI-27

Hawkins, Randy FU-2
Hayes, Laura GI-13, NE1-8, NE1-13, PNE-71

Hayward, Jessica PGI-59

Heaslip, Ingrid PMI-19

Heathcock, Jill PFN-25, PMI-31

Hebestreit, Helge EM-14

Hedrick, Holly FN-5

Hegde, Shilpa PCV-7, PGI-35, PMS-43

Hegde, Shilpa V. PMS-41

Heidema, Jojanneke PNE-28

Heim, Norbert MS2-R2

Hellinger, Jeffrey CH-14

Helm, Emma GU-6, PGU-20

Helmke, Knut PGI-20

Heng, Pa NE2-1

Henkel, David PNE-67

Herfkens, Robert EM-R1

Herliczek, Thaddeus PED-4, PGI-42, PGI-43

Hernandez Aceituno, Daniel GI-1

Hernandez, Ramiro PCV-7

Hernanz-Schulman, Marta GU-4

Herrmann, Jochen PGI-20

Hibbard, Roberta NA-5

Hicks, Ralph NA-5

Hicks, Rodney MI-3

Hilario, Amaya GI-1

Hilatrio, Amaya PGI-27

Hildebolt, Charles RS-R3

Hill, Jeanne PRS-14

Hill, Robert PRS-17, PRS-22

Hiller, Nurith FN-10

Hinds, Tanya NA-2

Hing, Anne PNE-33

Hintz, Susan PFN-28, PFN-15

Hirose, Shinjiro PGI-48

Hitchon, Patrick PNE-36

Ho-Fung, Victor PMS-59

Ho, PhD, Ngoc NE1-11

Hoban, Ciara PGI-25

Hofman, P PNE-26

Hoggard, Eric PGI-69

Holdsworth, Samantha NE1-R1,

PNE-65, PNE-66

Holland, Scott PNE-67

Holm, Tara PGI-54

Holmström, Miia PCV-2

Holubkov, Richard PGI-67

Hopkins, Katharine PRS-17, PRS-22

Hospach, Toni MS2-R2

Høising, Johan PRS-11

Hryhorczuk, Anastasia PGI-61

Hsiao, Albert CV-10, EM-R1
Huang, Chun-Chao PON-9

Huang, Jessica FU-1, FU-3

Huda, Walter PRS-14

Huelsemann, Wiebke PMS-30

Hughes-Roberts, Ynyr PMS-8

Hui, Peter PMS-21

Hui, Peter Kei Tat PMI-15

Huisman, Thierry PGI-47, PNE-48

Huisman, Thierry A.G.M. PGU-32

Huisman, Thierry AGM PNE-56

Hume, Jessica MS1-R1

Humphrey, Terry GI-9

Humphries, Paul FU-4, MS1-5, ON-5, PON-5

Hurwitz, Lynne RS-7

Husain, Aliya PGI-44

Husainy, Mohammad Ali PMI-16

Ibañez, Osvaldo PCH-24, PGU-34

Ieumsawasdikul, Kriengkrai PON-10

Ihn, Yonkwon PNE-1

Ilgan, Seyfettin PGU-4

Ilharreborde, Brice PMS-17

Iliadis, Kyriacos PCH-4

Iliadis, Kyriakos PCH-13

Imeri, Argjend RS-13

Inarejos, Emili PCH-11

Inarejos, Emilio PNE-14, PNE-18,

PON-21

Ingram, Mark NA-6

Ip, Janice PMI-15

Ip, Joana PON-11, PON-12

Iphantis, Nikolaos PCH-19, PGI-29,

PON-25

Irwin, Greg PGI-18

Ishak, Gisele NE1-7, PMS-36, PMS-37, PMS-38, PNE-32, PNE-33, PNE-41

Israel, Esther GI-4, GI-17

Israelis, Sara MS2-R1

Ito, Yoshiaki PCH-15

Ivey, Susan L NA-4

Iyer, Ramesh PMS-37, PMS-39,

PNE-32, PNE-33

Izci, Yusuf PNE-13

Jabs, Kathy GU-4

Jackson, Allison NA-2

Jackson, Dan PGI-67

Jackson, Michael MS2-3

Jacob, Joe PGI-21

Jacob, Joseph GI-11, PGI-5, PGI-22

Jacobson, Jeffrey FN-11

Jaeggi, Edgar CV-1 
Jaimes, Camilo PGU-37, PMS-62

Jain, Kajal MI-1

James, Julia PMI-20

Jamieson, Douglas PGU-31, PON-37

Jamuar, Saumya Shekhar PMS-11

Janitz, Emily GI-5

Jans, Lennart MS1-3

Jaramillo, Diego PFN-19, PMS-59,

PMS-64

Jaremko, Jacob MS1-3

Jarrett, Delma MS2-12, PMS-67

Jasinska, Aleksandra PGI-16

Jastrzebska, Malgorzata PNE-19

Jaya, Amarnath PMS-25

Jayarajasingam, Rubaraj PMI-11,

PNE-8

Jeanes, Annmarie GI-18, MS1-7,

PON-24

Jenney, Meriel PFN-5, PON-15

Jennings, S. Gregory NA-5, PMI-33,

PMI-36, PMS-34

Jeon, Tae Yeon NE1-5, ON-1, RS-4

Jeong, Hyun Yoo MS2-11

Jeong, Sujin PGU-3, PGU-13

Jin, Chunhua NE1-6

John, Philip IR-2, PIR-4, PIR-7, PMS-5

Johnson, Karl PMS-26

Johnson, Mark P FN-5

Johnson, Neil PMI-27, PMI-28, PMI-27

Jokinen, Eero PCV-2

Jones, Blaise NE1-2, PNE-67

Jones, Jeremy MS1-7, PGI-9

Jones, Richard GI-13, GU-2, NE1-8, PNE-71

Jones, Richard A NE1-13

Jong, Roland FU-5

Jordaan, Marc PNE-47

Jordaan, Marc R. PNE-54, PNE-55

Jose, Folashade PGI-62

Joshi, Aparna PFN-12

Josserand, Emilie FN-2

Jouannic, Jean-Marie FN-R2

Joubert, Ilse EM-5

Judy, Estroff PGI-66

Jung, Won Sang PNE-1

Kadom, Nadja PNE-61

Kaiser, Sylvie PRS-1, PRS-2

Kaitartzis, Christodoulos PMI-7, PNE-16

Kajbafzadeh, Abdol-Mohammad

PGU-19

Kaji, Yasushi PNE-2
Kajosaari, Merja PCH-6

Kalajoki-Helmiö, Teija PRS-7

Kalender, Willi A. RS-2

Kalra, Mannudeep CH-13

Kalra, Naveen PGI-15

Kalzen, Håkan PCV-1

Kamal Ahuja, Chirag PGI-10

Kampas, Ilias PGU-16, PMI-17

Kan, J. Herman GU-4, PMS-55

Kanavaki, Aikaterini PRS-6

Kaplan, Bernard PGU-37

Kappil, Mariam PGI-68

Kapur, Jeevesh PGU-5, PON-18

Karagiannidis, Ioakeim PCH-19,

PGI-29, PGU-22

Karakas, S Pinar PNE-63

Karakas, S. Pinar PNE-59

Karikoski, Riitta NE2-R1

Karmazyn, Boaz GU-11, NA-5,

PMI-33, PMI-36, PMS-58, PMS-63

Kashgari, Amna PMS-65

Kashou, Nasser PFN-25, PNE-69

Kassner, Andrea NE1-14

Kaste, Sue MS2-8, ON-8

Katarachias, Konstantinos PCH-14

Katelari, Anna PGU-11

Katsimba, Daphne PNE-16

Katz, Aubrey GI-4

Kaufman, Robert ON-8

Kaushal, Sunjay CV-3

Kaushik, Chhavi PNE-43

Kecskemethy, Heidi PGI-56

Keller, Frank ON-4

Keller, Marc CV-6

Kelliher, Emma PON-36, PON-38

Kelly, Teresa PNE-60, PNE-62

Kemp, Alison NA-1, NA-R2

Kenda, Rajko B GU-7

Kenney, Ian PMS-13

Kersbergen, K. FN-12

Kersnik Levart, Tanja GU-7, PIR-1

Kessler, Marcus RS-R3

Khandelwal, Niranjan CH-3, GI-6,

GI-10, MI-1, PGI-6, PGI-10

Khanna, Geetika PMS-42

Khanna, Paritosh PMS-36, PMS-37,

PMS-38, PMS-39, PNE-32, PNE-33,

PNE-41

Khong, Pek Lan PNE-6

Khurana, Aman FU-2

Khwaja, Asef PMS-46

Kiechl-Kohlendorfer, Ursula GU-1

Kiely, Edward EM-R2
Kilburn-Toppin, Fleur PCH-3, PMS-6, PMS-9, PON-8

Kim, In-One FN-8, MS2-5

Kim, Jane PGI-36

Kim, Jee-Eun PGU-9

Kim, Ji Hye NE1-5, ON-1, RS-4

Kim, Ji Young FN-8, MS2-5

Kim, Kp PRS-3

Kim, Martha Hanemann PFN-13, PGI-40, PNE-37, PNE-45

Kim, Stanley IR-5

Kim, Stanley T. CV-3

Kim, Woo Sun FN-8, MS2-5

Kim, Yoo Jin FN-8

King, Marta PGI-67

King, Susan PRS-10

Kingston, Kate PMS-32

Kirsch, Andrew GU-2

Kis M.D., Éva EM-7

Kissoon, Natalie NA-2

Kitano, Yoshihiro PIR-3

Kitchin, Douglas RS-R3

Kleinman, Paul MS2-12, NA-R1,

PMS-67

Kleinman, Paul K NA-4

Kline-Fath, Beth PFN-26, PON-34

Kline-Fath, Beth M. PFN-24

Kljucevsek, Damjana GU-7, PGI-8,

PGI-17, PIR-1

Kljucevsek, Tomaz PIR-1

Klokocovnik, Tomislav PIR-1

Klotz, Kerstin EM-1

Knudtson, John MS2-10

Ko, Hyun Soo PGI-63

Kocaoglu, Murat PGU-4

Koch, Bernadette PMI-27, PMI-28, PMI-27

Koerner, Markus PRS-9

Kohda, Ehiichi PCH-16

Köhler, Thorsten PMI-14

Kokorowski, Paul RS-14

Kondo, Mutsuko PCH-15

Kono, Tatsuo PCH-15

Kono, Tatuo PNE-10

Koob, Mériam PFN-6

Koplewitz, Benjamin PCH-21

Koronaios, Georgios PCH-14

Korostyshevskaya, Aleksandra PFN-2

Kortesniemi, Mika PRS-8

Kosnik, Edward PNE-70

Köstler, Herbert EM-14

Koumanidou, Chrysoula PGI-29,

PGU-22, PON-25 
Kourou, Evgenia PCH-19, PGU-22, PON-25

Koutsogianni, Ioannis PGI-29

Koutsogiannis, Ioannis PCH-19, PON-25

Kovanlikaya, Arzu PGI-59

Kowal, Alexander PGI-49, PON-32

Kowalska, Barbara PNE-19

Kozakewich, Harry MS2-14

Kraft, Jeanette MS2-R1

Kraft, Jeannette PMS-15, PMS-28

Kramer, Dennis PMS-67

Krasin, Matt FU-R1

Kring, Rune PRS-11

Krishna, Rama PCH-8, PCH-18, PNE-17

Krishnamurthy, Rajesh MS2-11,

PCV-9, PGU-38

Kritsaneepaiboon, Supika PMS-7,

PNE-7

Kumar, Praveen CH-3, GI-6

Kumar, Sumeet PMI-1

Kumbla, Surekha PMI-12, PNE-9,

PNE-12

Kurian, Jessica PGI-36

Kuroda, Tatsuo PIR-3

Kuwashima, Shigeko PCH-16, PNE-2

Kvanta, Eva PCV-1, PCV-6, PRS-1

Kwatra, Neha PNE-52

Kwon, Jung Yi NE1-5

Laborie, Lene B MS2-1

Laborie, Lene Bjerke MS2-2, MS2-6

Lacey, Stephen PNE-9

Ladino Torres, Maria F. PON-31

Ladino-Torres, Maria PGI-35

Laffan, Eoghan PMI-19, PMS-29

Lai, Jin-Yao PGI-12

Lai, Peng EM-11, PMS-44

Lai, Priscilla PRS-18

Lainez, Elena PNE-11

Lam, Wendy CV-8, PMS-21

Lam, Wendy Wai Man PMI-15

Lamminen, Antti PMS-12

Lancella, Laura PNE-25

Landes, Caren PGI-72, PGI-11, PMS-14, PMS-27, PON-13

Lane, Kathleen PGI-30, PGI-32, PGI-33

Langendörfer, Micha MS2-R2

Langston, Claire $\mathrm{CH}-1$

Lanza, Cecilia PFN-3, PGI-73, PGI-70, PON-6, PON-7

Lapierre, Chantale FN-6

Lara, Johnson RS-R2

Larson, David RS-1, RS-R2
Latini, Alessandro GI-8

Lattanzi, Ruggero PNE-24

Lauerma, Kirsi PCV-2

Laufer, Marc PGU-39

Lawrence, Tony PMI-25, PRS-15

Lawson, Zoe NA-1, PCH-1

Laxer, Ronald PMS-47

Lazda, Edgar FN-14

Le, Tam EM-13

Lederman, Henrique ON-6, PNE-72

LEDERMAN, HENRIQUE M PGU-27

Lee, Edward CH-7, PGI-50, PMS-7, PNE-7

Lee, Jee Hoon NE1-5

Lee, Joon PMS-13

Leenen, Andreas PMS-30

Lehmann, Trude G MS2-1

Lehmann, Trude G. MS2-2

Lehmann, Trude Gundersen MS2-6

Leitner, Maya NE2-2, PFN-23

Lenoch, Susan PED-3

Leong, Samuel PNE-23

Leopard, Catherine PMI-28

Lester, Neil FN-4, PFN-10

Leung, Jackie NE1-14

Leung, Vivian YF GI-3

Leung, Wing-Hang MS2-8

Leva, Ernesto PGI-71

Levin, Terry L PGI-34

Li, Cody NE2-2, PFN-23

Li, Hongtai PGI-48

$\mathrm{Li}$, Surong CH-R3

Li, Xiang RS-7

Li, Xinhua CH-13, RS-11

Li, Yimei ON-8

Liang, Xc CV-8

Lidegran, Marika PCV-1, PRS-1

Liechty, Kenneth CH-14

Lim, Foong-Yen PFN-24

Lim, Yun-Jung FN-8, MS2-5

Limbrick, David PNE-67

Linam, Leann PMI-23

Linam, W. Matthew PMI-23

Linnankivi, Tarja NE2-R1

Linscott, Luke RS-R3

Linsenmaier, Ulrich PRS-9

Lipsich, José PMI-32, PNE-64

Lisle, David MS2-9

Little, Stephen GU-2

Liu, Bob CH-13, RS-11

Livesey, Emily PGU-7

Lloyd, Claire PGU-31, PON-37

Lluch, Monica PGU-36
Lo Casto, Antonio PRS-4, PRS-5

Lo Zupone, Cristina GI-8

Lo, Wan-Chak PGI-12

Loder, Randall PMS-58

Loew, Wolfgang NE1-2

Loftis, Kathryn PFN-14, PMI-35

Loggitsi, Dimitra PGU-16, PMI-17

Lohi, Jouko PCH-6

Lomas, David EM-5, PMI-2

Long, Frederick $\mathrm{CH}-5$

Longo, Daniela NE1-9, PNE-25

López Gutierrez, Juan Carlos PMI-5

Love, MD, Terri EM-6

Lovrenski, Jovan $\mathrm{CH}-2$, CH-4

Low, David PIR-8

Low, Louis PMS-21

Lowe, Lisa PNE-49

Lowe, Lisa H. PED-1, PNE-38, PRS-15

Lubin, Jay PRS-3

Luisi, Flavio ON-6

Luo, Yu PGI-66

Lustig, Michael CV-10, EM-11, PMS-44

Ma, Benjamin PMS-66

Macgowan, Christopher CV-1

Mack, David EM-8

Macke, Jeremy PON-29

MacKinlay, Gordon A. IR-3

Madan, Shobhit CV-11

Maddu, Kiran PMS-43

Madzik, Jaroslaw ON-9, PNE-19

Maeder, Philippe NE2-4, ON-2

Magee, Fergall PFN-9

Maguire, Sabine NA-1, NA-R2

Mahan, Susan MS2-12

Maheshwari, Mohit PNE-60

Mahlmeister, Negar EM-1

Mahmood, Nadia PGU-38

Mahmood, Soran CH-7

Mahomed, Nasreen CH-9, PON-16, PON-17, PON-20

Mailhot, Johanna FN-R1

Mainwaring, Richard CV-R1

Majd, Massoud PNE-52

Mäkitie, Outi NE2-R1

Maley, Joan PED-3

Malghan, Lalita PON-19

Malik, Archana PGU-25

Malinen, Eirik NE1-4

Mall, Else-Gita PCH-20

Mallon, Mary PMS-53

Mallon, Mea PMS-49

Mallucci, Conor NE1-1 
Malone, Marian PGI-25

Man, Carina PMS-47

Manchanda, Smita PCH-7, PGU-15, PMI-13, PMS-23

Manchanda, Vivek PGU-15

Mandel, Keith RS-1

Mandell, Gerald FU-6

Mangano, Francesco PNE-67

Maniyar, Amit PCH-12

Mann, Erika PCH-2, PRS-19

Mann, Gurdeep PON-13

Mann, Mala NA-R2

Manson, David PCH-2, PCH-21, PON-30

Marabotto, Caterina PNE-25

Marchese, Paola PRS-4, PRS-5

Marin, Diana PMS-62

Marinaki, Eleni PCH-19, PGI-29,

PGU-22, PON-25

Maristany, Teresa PCH-11, PNE-14,

PNE-18, PON-21

Mark, Ferguson GI-14

Markowitz, Richard PRS-14

Marques, Vasco PON-11, PON-12

Marro, Kenneth GI-14

Marterer, Robert CV-4, CV-5, PCV-5

Martin Martinez, Cesar GU-9

Martin, Rose ON-10

Martinoli, Carlo MS1-1, MS1-2

Martus, Jefferey PMS-55

Maruskova, Linda MI-2, PMS-19

Masaki, Hidekazu PIR-3

Masand, Prakash PCV-9

Maurer, Kathrin GU-1, PNE-22

Mavridou, Charikleia PMI-7

Maximenko, Andrey FN-13

Mäyränpää, Mervi NE2-R1

Mazda, Keyvan PMS-17

Mazewski, Claire NE1-13

Mazzocchi, Silvia RS-5

Mbakaza, Mbaliso CH-9

McCarten, Kathleen ON-4, PGI-42

McCarter, Robert NA-2

McCarville, Beth FU-R1, PNE-58

McClean, Patricia GI-9

McDonald, Anna G NA-4

McGrane, Siobhan PNE-61

McGraw, Marty PMS-62

McGuire, Charles EM-12, PMI-26

McHugh, Kieran EM-R2, ON-7,

PCH-13, PGI-25, PRS-3

McIntosh, Adeka CV-6, PMS-64

McKenna, Christine PGI-64, PGI-65
McKinstry, Robert PNE-67, RS-R3

McLimont, Majorie MS2-R1

McMillan, MD, Jane NE1-11

McNeeley, Michael F. PIR-6

McNeil, Sarah PRS-18

McPhail, Gary EM-13

McPherson, Valerie ON-8

Mecozzi, Sara PON-22

Medeiros, Bruno Cunha PNE-46

Mehlman, Tracy PNE-59

Mehollin-Ray, Amy PGU-33

Mehta, Urmil GI-15, PGI-2

Melvin, Patrice PGI-61

Menchini, Laura PNE-25

Meneguzzi, Maria Belen PGU-35

Mentzel, Hans-Joachim PCH-20,

PMS-31

Meoded, Avner PNE-48

Mercer, Deanna PNE-67

Merina, Alicia PGU-18

Merinbaum, Debbie J. PNE-50

Merks, Johannes H.M. ON-7

Merlini, Laura PRS-6

Merrow, Arnold C. PFN-24

Metwalli, Zeyad CH-1

Meyers, Arthur PON-28

Meyers, Kevin PGU-37

Michael, Grattan CV-1

Michaud, Jean PNE-68

Midiri, Massimo PRS-4

Milesi-halle, Alessandra PNE-43

Miller, Elka EM-8, FU-5, PGI-51,

PNE-35, PNE-68

Miller, Jeffrey FU-6

Ming, Zhu CV-2, CV-7

Minnillo, Brian GU-3

Mino-Kenudson, Mari GI-4

Miron, Marie-Claude FN-6

Mishra, Michael CV-11

Mitchell, Christine FN-R1

Mitchell, Courtney PFN-28

Mitchell, Grace PGI-38

Mitchell, Sally PNE-56

Mittal, Vinayak GI-10

Miyazaki, Osamu PIR-3

Mizzi, Trent GI-2

Mody, Swati PFN-12

Moguillansky, Silvia PMI-32, PNE-64

Mohanta, Arun PNE-42

Mohsin, Arif PFN-25

Mohta, Anup PGU-15

Moineddin, Rahim FU-5, MS1-4

Mokhless, Ibrahim PGU-1, PGU-2
Moll, Annette C. NE2-4, ON-2

Monti, Lidia GI-8

Moorthy, Ima PFN-1

Morelli, John N. PMI-25, PNE-38, PNE-49

Morgan, Timothy PNE-49

Morio, Tomohiro Morio PCH-16

Morlese, John PNE-27

Morneault, Linda FN-9, NE2-3

Moroz, Paul MS2-7

Morris, Hayley NA-3

Morris, Sue NA-R2

Morris, Susan FN-14, NA-1, PCH-1

Mortilla, Marzia PNE-20, PNE-21

Moss, Helen PMS-8

Mosse, Yael PGU-37

Mouka, Vasiliki PGU-11

Moutard, Marie-Laure FN-R2

Muchart, Jordi PCH-11, PNE-14,

PNE-18, PON-21

Mulkern, Robert EM-10, FN-4

Mumraz, Hina PGI-6

Munden, Martha PGI-45

Munden, Marthe PGU-33

Mundorff, Michael PGI-67

Munkeby, Berit H NE1-4

MuÑoz Carpio, Veronica GI-1

Muñoz-Almagro, Carmen PCH-11

Murakami, James IR-4

Murakami, Terumi PCH-16

Murati, Michael PMI-34

Muridan, Roziah PGU-10

Muroya, Koji PNE-10

Murphy, Anna PMS-32

Murphy, Feilim PGU-17

Murphy, Mark CV-10

Mutch, Steven PRS-13

Nachman, James ON-4

Nadel, Helen EM-R3

Nagel, Bert CV-5, PCV-5

Nagel, Ed PMS-58

Naheedy, John PED-2, PNE-57

Naheedy, John H. PNE-54

Nakagawa, Motoo PFN-4

Nakamura, Olavo Kyosen PFN-13,

PGI-40, PNE-37, PNE-45, PNE-46

Nalpantidou, Chrysi PNE-16

Narang, Gurpreet PON-8

Nardo, Lorenzo PMS-66

Naser, Husain PON-14

Nathoo, Fatima NE1-14

Navarro, Oscar ON-R1, PCH-21, 
PMS-51, PMS-56, PNE-42

Naveed, Nausheen MS1-6

Neel, Michael MS2-8

Nees, Katherine PMI-27, PMI-28, PMI-27

Negus, Ian PRS-10

Negus, Samantha NA-6, PGU-17

Nelson, Caleb RS-14

Nelson, Michael PMI-34

Nemeth, Gabor PCH-17

Neramballi, Malini PNE-47

Ness, Kirsten MS2-8

Neuman, Jeremy PFN-24

Newbrough, Keith PMS-34

Newman, Beverley PCH-9, RS-10

Newman, Beverly CV-R1

Ngan, Bo-Yee PMS-56

Nguyen, Cheri PNE-44

Nguyen, Dustin PGU-30

Nguyen, Hiep GU-3

Nietosvaara, Yrjänä PMS-12

Nievelstein, R. FN-12

Nievelstein, Rutger A.J. FN-12

Nijs, Els PIR-8

Nimkin, Katherine GI-4, GI-17,

PGI-39, PMS-40

Nishikuni, Koshiro PNE-46

Nishimura, Gen PCH-15, PNE-10

Noccioli, Bruno PFN-8

Nogueira Delfino, Luciana NE1-9,

PNE-25

Nordenstam, Felicia PCV-1

Nordin, Mazin PGU-10

Nordlander, Maria MI-2

North, Kay PMI-25

Nosaka, Shunsuke PIR-3

Novack, Tom E. Novack PGU-32

Novell Teixido, Francesc GU-9

Novotny, Edward NE1-7

Ntai, Sparti PCH-19, PGI-29,

PGU-22, PON-25

Ntoulia, Katerina GU-8

Nyitrai M.D., Anna EM-7

O'Gorman, Molly PGI-67

O'Halloran, Rafael PNE-65

Oates, Adam PCH-21, PON-30

Obeid, Ibrahim PMS-17

Oberdabernig, Barbara RS-R1

Offiah, Amaka MS1-R1, NA-3

Ohsawa, Makiko PCH-16

Ojemann, Jeffrey NE1-7

Oligny, Luc PFN-16
Oliver, Jose-María PCV-4

Ording-Muller, Lil-Sofie PMI-14, PMS-24, PMS-25

Orentlicher, Rona PGI-34

Orman, Gunes PMI-6

Ortiz-Santiago, Madai PMS-59

Otto, Randolph GI-14

Oubel, Estanislao PFN-6

Oudjhane, Kamaldine PGI-63

Oury, Jean Francois FN-2

Owens, Catherine M PCH-4, PCH-5

Ozcan, Emrah PGU-4

Pacharn, Preeyacha PON-10

Padfield, Nancy PRS-19

Paesano, Pierluigi GU-R1

Pai, Deepa PMS-43

Pai, Deepa R. PMS-41

Pai, Mohan MS2-R1

Palagyi, Kalman PCH-17

Palasis, Susan NE1-8, NE1-13

Palko, William EM-12, PMI-26

Papachristou, Fotis GU-8

Papadakis, Antonis RS-9

Papadopoulou, Frederica GU-8

Papaioannou, Georgia PGU-16, PMI-17

Pappo, Alberto ON-8

Pariente, Danièle EM-2

Paritosh, Khanna NE1-7

Park, Ellen PNE-59

Park, Eunkyu PGU-3, PGU-13

Park, John PGI-57

Park, Kate PRS-13

Park, Soo PMI-36

Park, Won Soon NE1-5

Park, Young Nam PGU-9

Parker, Louise PRS-3

Parnell, Shawn PNE-33

Parra, Dimitri PIR-7

Parrón, Manuel PCV-4, PGI-13,

PGI-14, PMI-5

Parsons, Caron PGU-20

Pasquini, Lucia PFN-7, PFN-8

Pastor, Ignacio PGI-13

Patel, Amy PNE-49

Patel, Ketul PGI-21, PGI-22

Patel, Nirav PGI-72, PGI-11, PNE-5, PON-13

Patel, Preena GI-11

Patel, Suchit PED-1, PMI-25, PNE-49, PRS-15

Patil,, 2. Dr Aruna, PGU-14

Pattison, Sophie PON-5
Patton, Alice PNE-50

Pauly, John PMS-44

Paunovic, Milan PGU-8

Pavesi, Mariangela PGI-71

Pavicevic, Polina PGU-8

Pavicwic, Polina PGU-12

Pavlo, Jennifer PNE-40

Pazzaglia, Cristina PON-22

Peacock, Aurora PON-26

Pearce, Mark PRS-3

Peh, Wilfred PMS-1

Pei, Deqing MS2-8

Pellegrini, Maurizio PNE-24

Peltonen, Jari PMS-12

Pennison, Melanie RS-14

Penukonda, suvarna latha PGI-1

Pérez Vigara, Ana PGI-13, PGI-14, PMI-5

Perez-Atayde, Antonio PGI-50

Perez-Rossello, Jeannette NA-4

Pérez-Vigara, Ana PCV-3, PCV-4

Perez, Lizbet PGI-52

Perisinakis, Kostas RS-9

Perito, Emily PGI-62

Perkins, Susan PGI-30, PGI-32, PGI-33

Perreault, Gilles FN-6

Perrone, Anna PRS-12

Persaud, Thara PMS-24, PMS-25

Pérsico, Susana PNE-64

Peter, Mueller RS-11

Peter, Strouse J PMS-43

Peterson, Andrew PMI-30

Pettersson, David PRS-17, PRS-22

Pevsner Crum, Rachel PMS-60

Phillips, Timothy M. PGU-32

Piaggio, Lisandro PGU-35

Pierani, Paolo PON-22

Pieroni, Giovanni PFN-3, PGI-70, PON-22

Pierro, Agostino EM-R2

Pirani Esposto, Paolo PON-22

Pitkänen, Olli PCV-2

Pizer, Barry NE1-1

Pizzutillo, Peter PMS-54

Plata, Juan RS-10

Platt, Kaye PRS-13

Plumb, Andrew PGU-21

Podberesky-Alexander Towbin, Daniel

RS-1

Podberesky, Daniel CH-11, CH-12,

PGI-58, PMS-46, PON-28, RS-8

Pohl, Daniela PNE-68

Poletto, Erica PGI-34 
Poliakov, Andrew NE1-7

Pollock, Avrum N. EM-4, PNE-53

Polo, Luz PCV-4

Polsani, Aruna PON-35

Poon, Man-Chiu MS2-R1

Popescu, Andrada CV-3

Poretti, Andrea PNE-48

Porter, Neil PGU-21

Portia, Kreiger CH-14

Pose, Georgette PGI-52

Poskitt, Ken PNE-51

Postma, A PNE-26

Powell, Colin PCH-1

Pöyhiä, Tiina PMS-12

Prabhu, Sanjay PNE-47, PNE-57

Prabhu, Sanjay P. PNE-54, PNE-55

Prabhu, Somnath PIR-6

Prasad, Akhila PCH-7, PGU-15,

PMI-13, PMS-16, PMS-22, PMS-23

Pratt, Ronald NE1-2

Precht, Helle PRS-11

Price, Anita PON-36, PON-38

Prieto, Consuelo PCV-3, PGI-13,

PGI-14, PMI-5

Prince, Jeffrey RS-1

Prosser, Ingrid NA-1

Pruthi, Sumit PMS-36, PMS-37, PMS-38

Pryde, Scott PNE-56

Puccio, Roberta PRS-4, PRS-5

Punwani, Shonit FU-4, ON-5

Pupillo, Maria Clementina PMI-21

Qiu, Deqiang PNE-6

Qu, Hong NE1-4

Quadros, Marianne Siquara PFN-13,

PGI-40, PNE-37, PNE-45

Quattrocchi, Carlo PNE-25

Quattrocchi, Carlo Cosimo NE1-9

Quayle, Kimberly RS-R3

Quehenberger, Franz CV-4

Quencer, Keith GI-4

Rabinowitz, Deborah PIR-8

Raghavan, Deepak PGI-42

Raissaki, Maria RS-9

Rajagopal, Akila PNE-67

Rajkumar, Veena PIR-2

Rajpathak, Swapnil PFN-22

Rajwal, Sanjay GI-9

Rakhra, Kawan MS2-7

Ramamurthy, Satheesh PFN-5, PIR-2, PON-15

Ramanauskas, Fiona PMI-12, PNE-9
Ramsden, William PON-19

Randisi, Francesco NE1-9, PNE-25

Ranjan, Sanjeev PMS-16

Rao, Katragadda GI-10, PGI-7, PGI-15

Rao, Kln PGI-10

Rasalkar, Darshana D NE2-1

Rasalkar, Darshana Dattatray GI-3

Rasero, Miguel CH-6

Raspall, Miquel PNE-11

Raviv-Zilka, Lisa FN-11

Rayner, Tammy FU-5

Rebollo, Monica PCH-11, PNE-14, PNE-18, PON-21

Reddihough, Dinah NE1-R2

Reddy, Mohan CV-R1

Regacini, Rodrigo ON-6

Reichert, Cathy PMS-34

Reid, Janet PNE-63

Reid, Susan NE1-R2

Reilly, Gwen NA-3

Reiser, Maximilian PRS-9

Rentas, Kenny PED-1, PRS-15

Resteghini, Nancy PGI-37

Restrepo, Ricardo PMS-60

Retik, Alan GU-3

Reynolds, Ryan PED-3

Riaza, Lucia PCH-11, PNE-14,

PNE-18, PON-21

Ribo, Jose Luis PNE-14, PNE-18

Ribo, Josep PON-21

Ribó, Josep Lluís PCH-11

Riccabona, Michael RS-R1

Richmond, Joann M NA-4

Ridley, Lonay IR-R1

Riera Soler, Luis GU-9

Riera, Luis CV-4

Rigopoulou, Aspasia PGI-23

Rigsby, Cynthia GI-12, GI-19, PGI-68

Rigsby, Cynthia K. CV-3

Rivard, Georges MS2-R1

Rivas, Yolanda PGI-36

Rizzi, Ana PMI-32

Robben, Simon PNE-26

Roberts, Jeffrey H PGI-34

Robinson, Ashley PFN-11, PGU-31

Robinson, Denise FN-14

Robinson, Ian PMS-29

Rochester, Richard MS2-8

Rodjan, Firazia NE2-4, ON-2

Rodriguez Lemos, Ramón PGI-14

Rodriguez, Diana PNE-57

Roebuck, Derek PCH-13

Rogers, Marianne FU-5
Roik, Danuta PGI-16

Roman-Colon, Alicia PGI-45

Romaniuk-Doroszewska, Anna

PNE-19

Ron, Elaine PRS-3

Rosenberg, Alan PMS-33

Rosenberg, Andrew E NA-4

Rosendahl, Karen MS2-1, MS2-2,

MS2-6

Roth, Johannes PNE-68

Rougemont, Anne-Laure EM-3

Rowe, Courtney GU-3

Roymanee, Supaporn PNE-7

Rozhkova, Zinayida NE1-3

Rozovsky, Katya FN-10, PNE-35

Rubesova, Erika PFN-28, PFN-15

Rubio, Eva PFN-26, PON-34

Rubio, M PNE-26

Russo, Gianni GU-R1

Ryan, Martin PMI-18

Ryan, Stephanie PMI-19, PMS-29

Rypens, Françoise FN-6, PFN-16

Saarinen, Anne NE2-R1

Sachdev, Namrira PMS-22

Sachdev, Namrita PCH-7, PGU-15,

PMI-13, PMS-16, PMS-23

Saettele, Megan PED-1, PMI-25,

PNE-38, PNE-49, PRS-15

Saini, Deepak MI-1

Saint-Martin, Christine NE2-3

sainte-rose, Christian NE1-12

Saker, Martha PGI-68

Salahudeen, Safeeq NE2-2, PFN-23

Saleem, Sheena PFN-12, PNE-34

Salerno, Sergio PRS-4, PRS-5

Salisbury, Sheila RS-8

Salisbury, Shelia CH-12, ON-10, RS-R2

Salotti, Jane PRS-3

Salpietro, Daniela PNE-21

Samei, Ehsan RS-7

Sameshima, Yoshino Tamaki PFN-13, PGI-40, PNE-37, PNE-45, PNE-46

Sanchez-Montanez, Angel PNE-11

Sanchez, Esther ON-2

Sandhu, Ranbir PMI-9, PMI-10,

PMS-18, PMS-19

Sangkhathat, Surasak PMS-7

Sant'Anna, Guilherme FN-9, NE2-3

Santos-Campos, Xiomara PGU-38

Sarantidou, Kostantinia GU-8

Sargent, Michael PNE-51

Sarikwal, Anubhav MS2-9 
Sarsam, Shamil PCH-8, PCH-18, PNE-17

Sartor, Agnès FN-2

Sato, Takashi Shawn PMS-42, PNE-36

Sato, Yutaka PED-3, PNE-36

Savelli, Sara PFN-7, PFN-8, PNE-21

Saxena, Akshay CH-3, GI-6, GI-10,

MI-1, PGI-6, PGI-7, PGI-15

Saxena, Akshay Kumar PGI-10

Sayeed, Sadath FN-R1

Scherz, Hal GU-2

Schiavon, Jose ON-6

Schiavone, Raffaele PRS-12

Schicchi, Nicolò PMI-21

Schmit, Pierre GI-7, PFN-9, PMS-65

Schmitz, Kelli PRS-17

Schmitz, Kellie PRS-22

Schmitz, Matthew PMS-40

Schneider, Karl PRS-9, RS-3

Schnell, Beverly RS-R2

Schoot, Reineke A. ON-7

Schwartz, Cindy ON-4

Scoppetta, Luiz Raphael Pereira

Donoso PFN-13

Scott, Greig EM-11

Scrugham, Jeffrey PFU-3

Seah, Mayai MI-2, PMI-9, PMI-10,

PMS-18, PMS-19, PNE-28

Sebag, Guy FN-2, PMS-17

Sebire, Neil PCH-5, PNE-3, PNE-4

Seed, Mike CV-1

Segall, Hervey PNE-60

Segars, William Paul RS-7

Seibert, Joanna PMI-23

Seidel, F. Glen PFN-27, PGI-69

Seidenbusch, Michael RS-3

Sellars, Maria GI-11, PGI-5, PGI-21, PGI-22

Senes, Filippo MS1-1

Seow, Wan Tew PMS-11

Sepahdari, Ali PNE-57

Sera, Francesco MS2-2

Sera, Fransesco MS2-6

Serai, Suraj PON-28

Serrano, Carmelo GI-1, PGU-18

Servaes, Sabah PFN-19, PFU-2, PGI-44

Set, Pat EM-5, GI-15, PGI-2, PMS-8, PMS-9

Setty, Bindu N PGU-28, PGU-29

Seuri, Raija PRS-7, PRS-8

Sewing, A PNE-26

Seyed-Hossein Beigi, Reza PGU-19

Shafik, Mohamed PGU-2
Shaibani, Ali IR-1

Shaikh, Abdullah PGI-37, PGI-38, PGI-46, PGU-30, PMS-35, PNE-31

Shalaby-Rana, Eglal NA-2

Shammas, Amer FU-5, PCH-21, PIR-7

Shankar, Ananth FU-4, MS1-5, ON-5

Shankar, Samantha PON-27

Sharma, Shelly MS2-8

Sharp, Gregory PNE-43

Sharp, Susan FU-1, FU-3

Shaw, Dennis GI-14, NE1-7, PNE-32

Shaw, Dennis W. W. PIR-6

Sheahan, Deirdre CV-R1

Sheng, Ting-Wen PGI-12

Shenoy-Bhangle, Anuradha GI-17, PMS-40

Sher, Irene PGU-24

Shi, Lin NE2-1

Shibamoto, Yuta PFN-4

Shichiji, Minobu PCH-16

Shiels, William IR-4

Shifrin, Anna PNE-53

Shih, Shin-Lin PON-9

Shimony, Joshua PNE-67

Shin, Su-Mi FN-8

Shipman, Peter PNE-15

Shroff, Manohar NE1-14

Shulkin, Barry FU-R1

Sian, Aneet PGU-17

Siddiqui, Aslam PMI-33

Sidhu, Paul GI-11, PGI-5

Sigley, Matthew PNE-38

Silva, Cicero MS1-6

Silver, Ian NE2-2

Silvestri, Roberta GI-8

Simanovsky, Natalia FN-10

Simpkins, Carly PNE-15

Singh, Sarabjeet CH-13

Siomou, Ekaterini GU-8

Sion, Monica PNE-24

Sirikulvadhana, MPH, Laura NE1-11

Skalli, Wafa PMS-17

Skare, Stefan NE1-R1, PNE-65

Skidmore, David PFN-9

Skipper, Nicholas FU-4

Slaney, Charlotte PGU-6, PGU-7

Smal, Yulia RS-2

Smirniotopoulos, James PNE-30

Smith, Barry EM-8

Smith, Christopher PMI-25

Smith, Coral PGI-30

Smith, Ethan A. ON-10

Smith, Mark PMI-31
Smoljanic, Zeljko PGI-19, PGI-28

Smponia, Angeliki PNE-16

Snyder, Emma PNE-70

Soboleski, Don NE2-2, PFN-23

Sockolow, Robbyn PGI-59

Sodhi, Kushaljit CH-3, GI-6, GI-10,

PGI-6, PGI-7, PGI-10, PGI-15

Solaiyappan, Meiyappan PGU-32

Solomon, Aliza PGI-59

Soman, Salil NE1-R1, PNE-66

Sonik, Arvind EM-11

Sonnappa, Samatha PCH-5

Sorantin, Erich CH-2, CH-4, CV-4, CV-5, PCH-17, PCV-5, RS-6, RS-13, RS-R1

Soto Giordani, Gloria PGI-52

Soudack, Michalle FN-11

Southard, Richard PMS-52

Spadari, Raffaella PMI-21

Specchio, Nicola NE1-9

Speggiorin, Simone PCH-13

Spencer, Andrew PED-1, PRS-15

Spencer, Helen CH-10

Spencer, Samantha MS2-12

Spiegel, Miryam PCH-21

Spizzirri, Ana PGU-34

Sprigg, Alan MS1-R1

Spunt, Sheri FU-R1

Sreedhar, Gayathri CV-11

Sreedher, Gayathri PGI-64, PGI-65

Sreeharsha, Tirumani PGI-6

Srivastava, Raj PGI-67

Stäb, Daniel EM-14

Staib, Lawrence MS1-6

Stall, Alec ON-3

Stanwell, Joanna PGU-17

Stark, Dan PON-24

States, Lisa PMS-49, PMS-53

Stec, Andrew A. PGU-32

Stegmann, Joachim PMS-30

Stein, Nina Rodrigues ON-R1

Steinbach, Lynne PMS-66

Stenzel, Martin PCH-20, PMS-31

Stephens, Michael PMS-29

Stevens, Donna PRS-17, PRS-22

Stevenson, Kimberly PGI-56

Steward, Michael MS1-5

Stirling, Heather PGU-20

Stitzel, Joel PFN-14, PMI-35

Stockton, Emma IR-R1

Stojanovi, Sanja CH-2, CH-4

Støvring, Anne PRS-11

Strauss, Keith RS-1, RS-14 
Strouse, Peter PCV-7, PGI-35

Strouse, Peter J. PMS-41

Strouse, Peter J. PON-31

Stuart, Sam IR-R1

Stuber, Tina MS2-R2

$\mathrm{Su}$, Yingzi ON-R2

Suarez, Angela PGU-34

Sudigali, Venkat PCH-12, PNE-27

Sun, Binjian PNE-71

Sung, Edward PGU-28, PGU-29

Suñol, Mariona PON-21

Supakul, Nucharin PMI-33

Supakul, Tanya PMS-34

Suri, Sudha PGI-7

Sutton, Leslie EM-4

Swanson, Jonathan PNE-32

Swenson, David PGI-43

Swinson, Sophie GI-18, PMS-28,

PON-24

Swirsky, Stephen PMS-62

Szkudlinska-Pawlak, Sylwia PNE-19

Tadros, Sameh CV-11, PGI-64, PGI-65

Tahir, Bilal NA-5

Tahir, Nasim PON-19

Tan, Prof. Tan Ah PON-14

Tan, Zhi Yie Judith CH-8

Tanaanantarak, Pattama PMS-7, PNE-7

Tandoon, Jantima PON-10

Tang, Phua Hwee PMS-11, PON-14

Taragin, Benjamin PGI-36

Taragin, Benjamin H PGI-34

Taranath, Ajay PMS-5

Tartaro, Armando PNE-24

Taylor, Andrew PNE-3, PNE-4

Taylor, George PED-2, PGI-61

Taylor, Stuart FU-4

Tekes, Aylin PGI-47, PGU-32,

PNE-48, PNE-56

Tempia, Michela EM-3

Templeton, Peter PMS-28

Teo, Eu Leong Harvey PMI-1, PMS-1, PON-4

Teo, Harvey Eu Leong PMS-1

Teresa, Kelly PMS-61

Ternes, Tyler MS2-10

Terzaki, Katerina PCH-19, PGI-29,

PGU-22

Thacker, Paul PMS-45

Thakrar, Darshit GI-19

Thapa, Baburam GI-10, PGI-15

Thapa, Mahesh PMS-36, PMS-37,

PMS-38, PMS-39, PNE-32, PNE-33,
PNE-41

Thayyil, Sudhin PNE-3, PNE-4

Tholen, Peter PMS-30

Thomas, John GU-4

Thomas, Karen PRS-19

Thomas, Kristen PMS-45

Thompson, Lynn PIR-8

Thomson, Kimberley PNE-12

Thrall, James CH-13

Thyagiarajan, Manigandan PON-8

Thyagirajan, Manigandan PMS-6

Tilea, Bogdana FN-2

Tippett, Richard PON-5

Tkach, Jean NE1-2

To, Michael PMS-21

Toiviainen-Salo, Sanna NE2-R1

Tomà, Paolo GI-8

Tomazic, Mojca GU-7, PGI-17

Tombesi, Maria Marcela PFN-20,

PFN-21, PGU-35, PGU-36

Tomlinson, Christopher MS1-4

Toncheva, Greta CH-12, RS-7, RS-8

Torounidis, Elias PMI-7

Torre, Michele MS1-2

Torricelli, Maurizio PGI-71

Tostes, Vivian PNE-72

Towbin, Alex PGI-58, PON-28

Towbin, Alexander J. ON-10

Towbin, Richard FU-6, PMS-52

Tracy, Donald CH-7

Trask, John GI-7

Traubici, Jeff PCH-21

Treves, S. Ted PRS-21

Treves, Ted GU-5

Trottier, Luc PNE-42

Trout, Andrew T. PMS-41

Tsai, Andy GU-5

Tsifas, Sotirios PGI-23

Tsimara, Maria PGI-23

Tsota, Irini PGI-23

Tuna, Ibrahim PGI-34

Tung, Joanna PMS-21

Twomey, Eilish PMI-19, PMS-29

Ucar, María Elena PCH-24, PGU-34

Udayasankar, Unni PNE-59, PNE-63

Uliasz, Maria ON-9

Urbine, Jacqueline PCH-22, PGU-25,

PMS-54, PNE-30

Utrilla, Cristina PCV-3, PCV-4

Vachhani, Neil PNE-59

Vakaki, Marina PCH-19, PGI-29,
PGU-22, PON-25

Valeria Pedemonte, Valeria PGU-36

Valle, Maura MS1-1, MS1-2

van Rijn, Rick R. ON-7

Van Straaten, H. FN-12

Van Winkle, MD, Patrick Van Winkle NE1-11

Van Wyk, Matthys PON-17

van Wyk, Matthys J CH-15

VanDenKerkhof, Elizabeth NE2-2,

PFN-23

VanDyke, Rhonda EM-13

Várkonyi, Ildikó EM-7

Varvarigou, Anastasia PGI-23

Vasanawala, Shreyas CV-10, CV-R1,

EM-11, EM-R1, PMS-44

Vaughn, Jeff PMS-52

Vavasis, Gerasimos PGI-29, PGU-22, PON-25

Vazquez, Elida PNE-11

Venick, Robert PGI-57

Verbeke, Jonathan I.M.L. ON-2

Veres M.D., Gábor EM-7

Vergnani, Jason PIR-6

Verstraete, Koenraad MS1-3

Vibbard, MD, MsPH, Casey NE1-11

Victoria, Teresa FN-5

Vigevano, Federico NE1-9

Vo, Nghia Jack PIR-6

von Kalle, Thekla MS2-R2

Voss, Stephan EM-10, ON-4, PED-2, PFU-3

Vossough, Arastoo EM-4

Vucinic, Bojan PGI-19, PGI-28

Vujanic, Gordon FN-14

Vukadinovic, Vojkan PGU-8, PGU-12

Vural, Gulsah PGU-40

Wall, Corey PNE-33

Wallach, Michael PGI-42

Wallihan, Daniel PGI-58

Wallner, Claus-Peter PRS-9

Walters, Michele NA-R1

Walters, Sam MI-2, PMI-10, PMS-19, PNE-28

Walz, Dan PON-27

Wang, Chao-Jan PGI-12

Wang, Defeng NE2-1

Wang, Jackson MS1-4

Wanner, Matthew NA-5, PMI-36

Wansapura, Janaka EM-13

Ward, Leanne MS2-7

Ward, Valerie GU-R2, PGU-39 
Warfield, Simon EM-10

Watson, Debbie PMI-8

Watson, Elizabeth PGI-59

Watson, Tom MS1-7, PGI-9, PMS-15,

PON-24

Weber, Alfred L PON-23

Weber, J PNE-26

Weinberger, Ed GI-14

Weingertner, Anne-Sophie PFN-6

Weiss, Amanda PFN-22

Weiss, Ruth FU-5

Weiß, Sandra EM-14

Weissensteiner, Sabine RS-13

Weldon, Christopher CH-7

Wells, Toby PGI-4

Wendel, John EM-6

Westra, Sjirk CH-13, PMI-24, RS-1

Wheelock, Lisa EM-6

Whitehead, Kevin CV-6

Wiebe, Sheldon PMS-33

Wiegering, Verena EM-1

Wilkes, David ON-3

Wilkinson, Alastair MS2-4, PMS-20

Wilkinson, Alastair Graham MS2-3

Wilkinson, Alistair Graham IR-3

Wilkinson, Sally MS2-3

Willamo, Patrick PMS-12

Williams, Alexandra PGI-72, PGI-11,

PNE-5, PON-13

Williams, Bhanu PMI-10

Williams, Suzan NE1-14

Willis, Baxter MS2-7

Wilson, Matthew W PNE-58

Wilson, Nagwa EM-8, PGI-51, PNE-68

Win, Thuzar PGI-3, PMI-11, PNE-8

Winkler, Peter MS2-R2

Wirth, Clemens EM-1, EM-14

Wlasdorff, Michel FN-7

Wong, Adelaine PMS-33

Wong, Derek CV-1

Wong, Sj CV-8

Woodley, Helen GI-9, PGI-9

Wozniak, Wojciech ON-9

Wright, Neville PGU-21

$\mathrm{Wu}$, Jianrong FU-R1

Wu, John MS2-R1

$\mathrm{Wu}$, Shuochun GI-16

Xiao, Jiangxi NE1-6

Xiaowei, Zhu CH-14

Xie, Sheng NE1-6

Yacobozzi, Margaret PNE-31
Yadav, Tribhuvan PMS-16

Yaddanapudi, Kavitha PNE-63

Yalynska, Tetyana FN-13

Yamane, Yusuke PIR-3

Yamashita, Kyou PFU-1

Yan, Yuchung CH-5

Yang, Fei-Shih PON-9

Yang, Jie MS2-8

Yang, Shengping MS2-8

Yee, Brian ON-8

Yemets, Illya FN-13

Yeom, Kristen PFN-28, NE1-R1,

PNE-65, PNE-66

Yeon, Kyung Mo FN-8, MS2-5

Yershova, Yevgeniya FN-13

Yilmaz, Sabri MS2-14

Ylitalo, Pekka PCV-2

Yoo, Shi-Joon CV-1

Yoo, So-Young NE1-5, ON-1, RS-4

Yoshizumi, Terry CH-12, RS-8

Yoshizumi, Terry T. RS-7

Youssif, Mohamed PGU-1, PGU-2

Yu, Chih-Hsiang PNE-44

Yuan, Weihong PNE-67

Yuan, Xinyu CH-R3, GI-16, NE1-6,

ON-R2

Yunus, Rohaizan PCH-2

Zahari, Zakaria PCH-10

Zahran, Abdelrahman PGU-2

Zand, Tristan PRS-6

Zarifi, Maria PCH-14, PGU-11

Zatelli, Giovanna RS-5

Zhong, Anguo FU-5

Zhou, Xiaodong RS-7

Zhu, Xiaowei RS-12

Zimmerman, Donald IR-5

Zimmerman, Robert A. EM-4, PNE-53

Zou, Maggie PRS-10

Zumberge, Nicholas PNE-39, PON-29

Zurakowski, David CH-7 